\title{
TITLE:
}

\section{Giant Nernst and Hall effects due to chiral superconducting fluctuations}

$\operatorname{AUTHOR}(S)$ :

Sumiyoshi, Hiroaki; Fujimoto, Satoshi

CITATION:

Sumiyoshi, Hiroaki ...[et al]. Giant Nernst and Hall effects due to chiral superconducting fluctuations. Physical Review B 2014, 90(18): 184518.

ISSUE DATE:

2014-11-10

URL:

http://hdl.handle.net/2433/193275

RIGHT:

(C)2014 American Physical Society 
PHYSICAL REVIEW B 90, 184518 (2014)

\title{
Giant Nernst and Hall effects due to chiral superconducting fluctuations
}

\author{
Hiroaki Sumiyoshi ${ }^{1}$ and Satoshi Fujimoto ${ }^{1,2}$ \\ ${ }^{1}$ Department of Physics, Kyoto University, Kyoto 606-8502, Japan \\ ${ }^{2}$ Department of Materials Engineering Science, Osaka University, Toyonaka 560-8531, Japan \\ (Received 17 March 2014; revised manuscript received 10 November 2014; published 24 November 2014)
}

\begin{abstract}
We consider the Nernst and Hall effects in the fluctuation regime of chiral superconductors above transition temperatures, that are raised not by the conventional Lorentz force but by a mechanism that is an analog of the anomalous Nernst or Hall effects, i.e., asymmetric scattering due to chiral superconducting fluctuations. It is found that these effects can be gigantic for cleaner samples compared to conventional ones, exhibiting qualitatively distinct behavior. The results provide systematic and comprehensive understanding for recent experimental observations of the Nernst effect in a clean $\mathrm{URu}_{2} \mathrm{Si}_{2}$ sample, which is suggested to be a chiral superconductor.
\end{abstract}

DOI: 10.1103/PhysRevB.90.184518

\section{INTRODUCTION}

In a certain class of superconductors, fluctuations toward ordered states above transition temperatures give rise to dramatic effects on many-body electron states. It is known that a powerful probe for such phenomena is the Nernst effect [1,2]. In ordinary metals in the normal state, the Nernst signal is generally weak owing to the Sondheimer cancellation $[3,4]$. Thus, a large Nernst signal near and above transition temperature $T_{c}$ implies superconducting fluctuation contributions such as short-lived Cooper pairs [5] and Josephson electromotive force due to the vortex motion $[6,7]$.

In this paper, we propose that a quite distinct mechanism of the giant Nernst and Hall effects is possible in clean samples of chiral superconductors, where time-reversal symmetry (TRS) is spontaneously broken and total angular momentum carried by Cooper pairs is nonzero. In chiral superconductors, below $T_{c}$, the intrinsic magnetic field induced by Cooper pairs with relative angular momentum, i.e., chirality, causes exotic transverse transport phenomena under zero external magnetic field, such as the Kerr effect [8,9], which was observed in $\mathrm{Sr}_{2} \mathrm{RuO}_{4}$ [10], and the anomalous thermal Hall effect, which was theoretically predicted [11-13]. It is natural to expect that also in the superconducting fluctuation regime above $T_{c}$, characteristic transverse transport phenomena can be induced by fluctuations of the chiral Cooper pairs. We investigate this possibility and clarify a mechanism of the anomalous Nernst and Hall effects above and near $T_{c}$, caused by chiral superconducting fluctuation (CSF). In this scenario, quasiparticles are scattered asymmetrically by fluctuating Cooper pairs with angular momentum, even without Lorentz force, and then such effects can be regarded as an analog of the skew-scattering process of the anomalous Hall effect, which is caused by a spin-orbit coupling involving impurity scattering [14], but a major difference is that the scattering kernels are dynamical in this case.

There are several candidate systems for chiral superconductors such as $\mathrm{Sr}_{2} \mathrm{RuO}_{4}$ and $\mathrm{URu}_{2} \mathrm{Si}_{2}$ [15-23]. Among them, the heavy-electron superconductor $\mathrm{URu}_{2} \mathrm{Si}_{2}$, whose pairing symmetry is suggested to be chiral $d_{z x} \pm i d_{z y}$ [16-18], is one of the most promising systems for the realization of the above-mentioned mechanism, because, for this system, strong superconducting fluctuation effects have been experimentally observed, which may be attributed to small energy scale raised by heavy effective mass and the reconstruction of electronic
PACS number(s): 74.25.fc, 74.20.-z, 74.62.-c, 74.70.Tx

structures in the so-called hidden order phase [24]. Thus, in this paper, we mainly focus on this system, though our theory is also applicable to other chiral superconductors such as $\mathrm{Sr}_{2} \mathrm{RuO}_{4}$ with minor modifications.

The organization of this paper is as follows. The model system mainly considered in this paper is given in Sec. II. In Sec. III, the results of the Nernst and Hall effects which are raised by asymmetric scattering due to chiral Cooper pairs are presented. We discuss some important features of these results, such as the dependence of the conductivity tensors on the relaxation time of quasiparticles and temperature, in Sec. IV. Implications of our results for experiments are given in Sec. V. We also consider the case of $\mathrm{Sr}_{2} \mathrm{RuO}_{4}$ in Sec. VI. In Sec. VII, we discuss paramagnetism induced by chiral superconducting fluctuations. Our summary is given in Sec. VIII.

\section{METHOD AND MODEL}

Our approach is based on microscopic model calculations utilizing linear response theory. The Hall conductivity is given by the Kubo formula. On the other hand, for the Nernst effect, one needs to take account of contributions from magnetization $\boldsymbol{M}$ in addition to those from the Kubo formula [25-28]. Then, the Nernst conductivity is $\alpha_{\alpha \beta}=\alpha_{\alpha \beta}^{\text {Kubo }}+\alpha_{\alpha \beta}^{\mathrm{mag}}, \alpha_{\alpha \beta}^{\mathrm{mag}}=$ $\epsilon_{\alpha \beta \gamma} M^{\gamma} / T$. Here, $\alpha_{\alpha \beta}^{\text {Kubo }}$ is the Kubo term given by the heat current-charge current correlation function.

The Hamiltonian with which we start is an effective model for the superconducting state of $\mathrm{URu}_{2} \mathrm{Si}_{2}$, with the pairing symmetry of the $E_{g}$ representation of the point group $D_{4 h}$ [29]:

$$
\begin{aligned}
\mathcal{H}= & \sum_{\boldsymbol{k} \sigma} \xi_{\boldsymbol{k}} c_{\boldsymbol{k} \sigma}^{\dagger} c_{\boldsymbol{k} \sigma}-g \sum_{\boldsymbol{k}, \boldsymbol{k}^{\prime}, \boldsymbol{q}} V\left(\boldsymbol{k}, \boldsymbol{k}^{\prime}\right) c_{\boldsymbol{k}+\frac{q}{2} \uparrow}^{\dagger} \\
& \times c_{-\boldsymbol{k}+\frac{q}{2} \downarrow}^{\dagger} c_{-\boldsymbol{k}^{\prime}+\frac{q}{2} \downarrow} c_{\boldsymbol{k}^{\prime}+\frac{q}{2} \uparrow},
\end{aligned}
$$

where, for simplicity, we take a spherical Fermi surface, $\xi_{k}=$ $\boldsymbol{k}^{2} / 2 m-\mu$, and $V\left(\boldsymbol{k}, \boldsymbol{k}^{\prime}\right)=15\left(k_{z} k_{x} k_{z}^{\prime} k_{x}^{\prime}+k_{z} k_{y} k_{z}^{\prime} k_{y}^{\prime}\right) / k_{F}^{4}$ is the effective pairing interaction in the $d_{z x}$ and $d_{y z}$ channels. It is the model for the chiral $d_{z x} \pm i d_{z y}$ superconducting state. In the chiral superconducting phase, TRS is spontaneously broken and the gap function takes the form $\Delta(\boldsymbol{k}) \propto k_{z}\left(k_{x}+i k_{y}\right)$ or $\left[k_{z}\left(k_{x}-i k_{y}\right)\right]$, which is caused by an effective attractive interaction, $V^{+}\left(\boldsymbol{k}, \boldsymbol{k}^{\prime}\right)=\phi(\boldsymbol{k}) \phi^{\dagger}\left(\boldsymbol{k}^{\prime}\right)$ or $\left.V^{-}\left(\boldsymbol{k}, \boldsymbol{k}^{\prime}\right)=\phi^{\dagger}(\boldsymbol{k}) \phi\left(\boldsymbol{k}^{\prime}\right)\right]$, where the pairing symmetry function reads $\phi(\boldsymbol{k})=\sqrt{15 / 2} k_{z}\left(k_{x}+i k_{y}\right) / k_{F}^{2}$. Note that 
$V\left(\boldsymbol{k}, \boldsymbol{k}^{\prime}\right)=V^{+}\left(\boldsymbol{k}, \boldsymbol{k}^{\prime}\right)+V^{-}\left(\boldsymbol{k}, \boldsymbol{k}^{\prime}\right)$. The channel $V^{+(-)}$is associated with the chirality $C=+1(-1)$, and each channel breaks TRS. However, we concentrate on transport phenomena above $T_{c}$, in the fluctuation regime, where two channels are degenerate, and therefore TRS is not spontaneously broken.

\section{NERNST AND HALL EFFECTS}

Generally, to induce transverse transport phenomena such as the Nernst and Hall effects, it is necessary to break TRS. In fluctuation regimes above $T_{c}$, TRS is not spontaneously broken, and then a magnetic field is necessary to break TRS. Due to a magnetic field, the Lorentz force on quasiparticles and fluctuating Cooper pairs is generated and causes conventional transverse transport phenomena [30]. In addition, in the case of chiral superconductors, the magnetic field also causes "polarization" of chirality due to a magnetic field-chirality (MC) coupling; i.e., the difference in the weights of two superconducting fluctuation channels is induced. The chiralitypolarized superconducting fluctuations give rise to asymmetric scattering of electrons resulting in the anomalous Nernst and Hall effects (ANE and AHE) without Lorentz force, which are the main subjects of this paper [see Eqs. (4)-(6) below, which constitute the main results].

First, we discuss the chirality polarization by evaluating the superconducting fluctuation propagator. Under a uniform magnetic field $\boldsymbol{H}=(0,0, H)$, the fluctuation propagators of chiral $d_{z x} \pm i d_{z y}$ channels (correspond to $C= \pm 1$, respectively) is given by (the derivation is described in Appendix A)

$$
\tilde{L}_{C}^{-1}\left(\boldsymbol{x}, \boldsymbol{y}, \omega_{q} ; H\right)=-\frac{\delta(\boldsymbol{x}-\boldsymbol{y})}{g}+\tilde{\Pi}_{C}\left(\boldsymbol{x}, \boldsymbol{y}, \omega_{q} ; H\right),
$$

where $\tilde{\Pi}_{C}\left(\boldsymbol{x}, \boldsymbol{y}, \omega_{q} ; H\right)$ is the bare particle-particle susceptibility (BPS), which is decomposed into the chirality-independent term [the first term of Eq. (3)] and the chirality-dependent term [the second term of (3)]:

$$
\begin{aligned}
\tilde{\Pi}_{C}\left(\boldsymbol{x}, \boldsymbol{y}, \omega_{q} ; H\right)= & e^{-i 2 e \Phi(\boldsymbol{x}, \boldsymbol{y})}\left[\Pi\left(\boldsymbol{x}-\boldsymbol{y}, \omega_{q} ; H\right)\right. \\
& \left.-C \frac{5 e H}{4 k_{F}^{2}} \Pi^{\prime}\left(\boldsymbol{x}-\boldsymbol{y}, \omega_{q} ; H\right)\right],
\end{aligned}
$$

where $\Pi$ and $\Pi^{\prime}$ are "core" bare BPSs which preserve translation, gauge, and $c$-axis rotation invariances [31-33], $\omega_{q}$ is the bosonic Matsubara frequency, and the phase, $\Phi(\boldsymbol{x}, \boldsymbol{y})=$ $\int_{\boldsymbol{x}}^{\boldsymbol{y}} \boldsymbol{A}(\boldsymbol{r}) d \boldsymbol{r}$, is defined as an integral of the vector potential along a straight line. The precise expressions of $\Pi$ and $\Pi^{\prime}$ are given in Appendix A. Note that this expression (3) is applicable to arbitrary magnitude of magnetic fields and for any gauge conditions. The remarkable point of (3) is that the amplitude of the BPS is changed by the MC coupling via the chirality-dependent term, $-C\left(5 e H / 4 k_{F}^{2}\right) \Pi^{\prime}$. As a result, the $\mathrm{MC}$ coupling raises (lowers) the transition temperature of the $C=-1(+1)$ state, which has orbital magnetic moment parallel (antiparallel) to the $c$ axis, in contrast to the phase $\Phi$, which reflects the orbital depairing effect, and always lowers the transition temperature [34]. Moreover, the MC coupling induces paramagnetism, discussed later.

Using the fluctuation propagator, Eq. (2), we calculate the Nernst and Hall conductivities. Note that up to the linear order

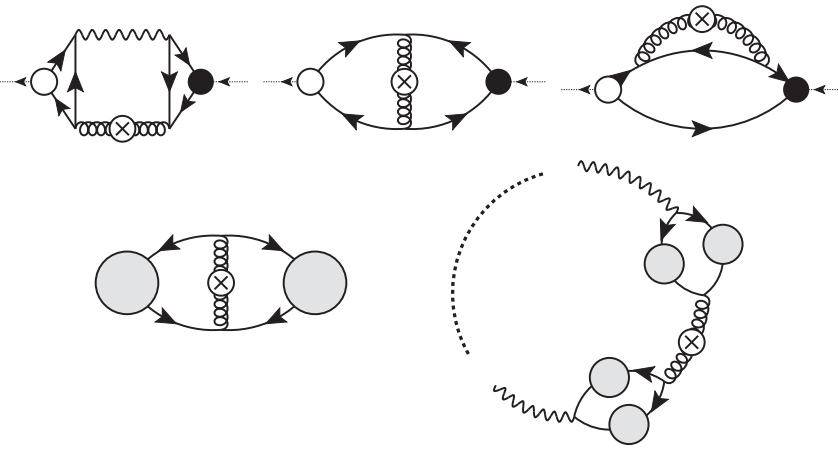

FIG. 1. Upper panel: AL, MT, and DOS diagrams. The AL and DOS diagrams have the mirror image counterparts. Wavy lines and curly lines with crossed circles represent the fluctuation propagator in zero magnetic field, $L$, and the chirality-polarized one, $\tilde{L}_{C}^{\prime}$, respectively, where their definitions are given in Appendix B. Solid lines with arrows are the one-particle Green's functions. Open circles represents electric current vertex, and bullets represent energy current vertex (electric current vertex), for $\alpha_{x y}\left(\sigma_{x y}\right)$. Lower panel: Diagrams in which the information of the chirality disappears. Shaded circles represent any diagrams without fluctuation propagators and the two current vertices are inserted into any propagators.

in $H$, we can systematically separate whole contributions into two parts: one corresponding to the conventional contribution due to Lorentz force on quasiparticles and fluctuating Cooper pairs, and the other one associated with the ANE and AHE caused by asymmetric scattering due to CSF. As will be shown below, the latter contribution dominates over the former one for clean samples. Thus, we focus on the latter in the following. We sketch briefly a basic idea of the derivation for the Nernst and Hall conductivities (see Appendix B for the details). It is found that the three diagrams which give leading-order contributions in conventional theories, i.e., the AslamazovLarkin (AL), Maki-Thompson (MT), and density-of-states (DOS) diagrams (upper panel in Fig. 1) [30], do not contribute in the absence of Lorentz force, and generally, all contributions from diagrams belonging to the classes of the lower panel in Fig. 1 are zero. The reason is that the cancellation of skew scattering occurs between electrons and holes (the details in Appendix B). The lowest order diagrams which do not belong to these classes and give nonzero contributions are depicted in Fig. 2. In these diagrams, scattering processes due to electronelectron interaction represented by a renormalized four-point (a)

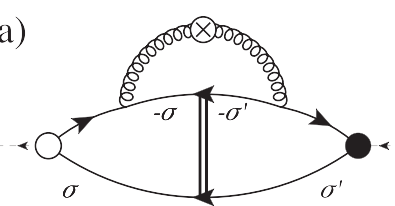

(c)

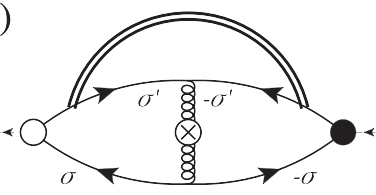

(b)

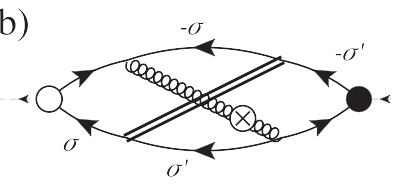

+ (Mirror Images)
FIG. 2. Diagrams which contribute to the ANE and AHE raised by the CSF mechanism. The double lines represent the renormalized four-point vertex, $W\left(\boldsymbol{k}, \omega_{j}\right)$. 
vertex, $W\left(\boldsymbol{k}, \omega_{j}\right)$ (double line), which disturb the abovementioned cancellation of skew-scattering, are included. To carry out calculations explicitly, we postulate a simple model: $W\left(\boldsymbol{k}, \omega_{j}\right)=W_{0} /\left(1+\left|\omega_{j}\right| / \Gamma\right)$, i.e., an interaction mediated via a short-range antiferromagnetic spin fluctuation, where $W_{0}$ is a constant and $\Gamma$ is the energy scale of spin fluctuations. In fact, for $\mathrm{URu}_{2} \mathrm{Si}_{2}$, a short-range antiferromagnetic spin fluctuation exists in the hidden order phase as clarified by inelastic neutron scattering measurements $[35,36]$. Thus, the above assumption for $W\left(\boldsymbol{k}, \omega_{j}\right)$ is legitimate. However, we stress that our final results are qualitatively not changed by the specific form of $W\left(\boldsymbol{k}, \omega_{j}\right)$, as will be discussed later.

Then, we obtain the Kubo terms of the Nernst and Hall conductivities in the clean limit, near $T_{c}$, and in the linear order of $H$ (the details of the calculation are described in Appendix B):

$$
\begin{aligned}
\frac{\alpha_{x y \text { chiral }}^{\text {Kubo }}}{H} & =\frac{f\left(\frac{2 \pi T}{\Gamma}\right)}{2304} \frac{\tau^{2} e^{2} W_{0} v_{F}^{4} \Lambda}{\xi^{4} g k_{F}^{2} T^{2}}\left(1-\frac{3 \pi}{4} \frac{\sqrt{\varepsilon}}{\xi \Lambda}\right), \\
\frac{\sigma_{x y \text { chiral }}}{H} & =\frac{5 f\left(\frac{2 \pi T}{\Gamma}\right)}{1152} \frac{\tau^{2} e^{3} W_{0} v_{F}^{3} \Lambda}{\xi^{4} g k_{F}^{3} T}\left(1-\frac{3 \pi}{4} \frac{\sqrt{\varepsilon}}{\xi \Lambda}\right) .
\end{aligned}
$$

Here, $\varepsilon=\log T / T_{c}, \quad v_{F}$ is the Fermi velocity, $\xi=$ $\sqrt{-\psi^{\prime \prime}(1 / 2) / 6}\left(v_{F} / 4 \pi T\right)$ is the coherence length, $\psi$ is the digamma function, $\tau$ is the electron scattering time due to impurities and electron-electron scattering, $\Lambda$ is the cutoff of the momentum of the superconducting fluctuation propagator, which is the same order as $1 / \xi$, and $f(2 \pi T / \Gamma)$ is a dimensionless function, whose definition and numerical estimations are given in Appendix D.

Now, we discuss the magnetization contribution. The magnetization due to chirality-polarized superconducting fluctuations is of interest not only because of its contribution to the Nernst effect, but also because of its unique magnetic property; i.e., the polarization of CSF causes paramagnetism in contrast to diamagnetism due to fluctuating Meissner currents observed in general superconductors [30]. The calculation is performed with the free energy of chiral superconductors above $T_{c}: F[H]=T \sum_{\omega_{q}, C= \pm 1} \operatorname{Tr} \ln \left[-\hat{\tilde{L}}_{C}^{-1}\left(\omega_{q} ; H\right)\right]$, where $\hat{\tilde{L}}_{C}^{-1}\left(\omega_{q} ; H\right)$ is the matrix whose indices are spatial coordinates, $\boldsymbol{x}$ and $\boldsymbol{y}$, and matrix elements are given by Eq. (2). From this free energy, we obtain the magnetic susceptibility $\chi=$ $\chi_{\text {dia }}+\chi_{\text {chiral }}$ (Appendix E), where $\chi_{\text {dia }}$ is the diamagnetic term due to fluctuating Meissner currents observed in general superconductors $[30,37]$, and $\chi_{\text {chiral }}$ is the paramagnetism term mentioned above. Then, the magnetization current contribution inherent in chiral superconductors is

$$
\frac{\alpha_{x y \text { chiral }}^{\mathrm{mag}}}{H}=\frac{\chi_{\text {chiral }}}{T}=\frac{25 e^{2}}{64 \pi k_{F}^{4} \xi^{3}[N(0) g]^{2} \varepsilon^{1 / 2}} .
$$

The total anomalous Nernst conductivity due to CSF is given by the sum of Eqs. (4) and (6), which constitute our main results.

\section{DISCUSSIONS OF EQUATIONS (4), (5), AND (6)}

We now discuss several important features of Eqs. (4) and (6). The critical behavior of the magnetization current

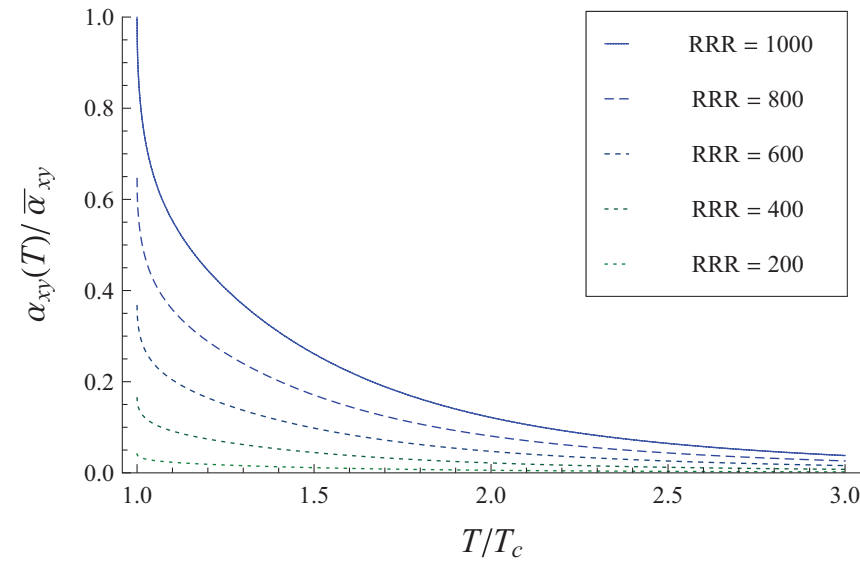

FIG. 3. (Color online) $\alpha_{x y}$ raised by the CSF mechanism versus $T / T_{c}$ for several values of RRR. The magnitudes of $\alpha_{x y}$ are normalized by the value of the most clean one at $T_{c}, \bar{\alpha}_{x y}:=$ $\alpha_{x y}\left(T_{c} ; \mathrm{RRR}=1000\right)$. We used the material parameters of $\mathrm{URu}_{2} \mathrm{Si}_{2}$.

contribution, (6), given by $\propto\left(T-T_{c}\right)^{-1 / 2}$, is the same as that of the AL term of the Nernst conductivity [5,38]. On the other hand, the critical behavior of the Kubo contribution, (4), is less singular, $\propto$ (const. $-\sqrt{T-T_{c}}$ ). However, we note that the dependence on scattering time $\tau$ of Eq. (4), which is proportional to $\tau^{2}$, is quite distinct from any fluctuation-induced corrections to the Nernst coefficient previously studied so far. For instance, there is no $\tau$ dependence in the contribution to $\alpha_{x y}$ obtained by dynamics of boson fields (i.e., fields of Cooper pairs), such as the scenarios of short-lived Cooper pairs [5] and the vortex motion [6]. This is simply because that dynamics of bosons do not involve quasiparticle scattering time. Also, it is known that contributions from electron dynamics influenced by the fluctuation boson field, including the MT and DOS terms, do not yield $\tau$-dependent $\alpha_{x y}[39,40]$. Thus, for sufficiently clean samples with large $\tau$, the Kubo term $\alpha_{x y \text { chiral }}^{\text {Kubo }}$ of the CSF mechanism significantly dominates over the conventional Nernst conductivity raised by Lorentz force. Also, because of the $\tau$ dependence, $\alpha_{x y \text { chiral }}^{\text {Kubo }}$ is much more enhanced than the magnetization term (6) for cleaner samples. Thus, the leading term of the Nernst conductivity for clean chiral superconductors is given by $\alpha_{x y \text { chiral }}^{\text {Kubo }}$. The unusual $\tau$ dependence of $\alpha_{x y \text { chiral }}^{\text {Kub }}$ combined with an increasing behavior for $T$ approaching $T_{c}$, as shown in Eq. (4), characterizes the distinct feature of the CSF mechanism. In Fig. 3, we plot typical temperature dependencies of Eq. (4) for several values of $\tau$ parametrizing the residual resistivity ratio (RRR) of samples. Here, we used material parameters of $\mathrm{URu}_{2} \mathrm{Si}_{2}$, and the calculation was achieved by using an approximation scheme explained in Appendix F. In Fig. 3, $\alpha_{x y}$ exhibits remarkably strong enhancement in the vicinity of $T_{c}$ for cleaner systems. It is an intriguing feature to test our theory for real materials. On the other hand, the Hall conductivity, Eq. (5), has the same characteristic $\tau$ dependence, $\propto \tau^{2}$, as $\alpha_{x y \text { chiral }}^{\text {Kubo }}$, and, moreover, is nonzero even when the electronic band is particle-hole symmetric. This point is quite different from conventional contributions derived from the time-dependent Ginzburg-Landau equation or, equivalently, the AL term [41]. However, it would be rather more difficult to detect the Hall 
effect than the Nernst effect, because normal Hall currents of Fermi-liquid quasiparticles dominate for charge transport.

The $\tau$ dependence of (4) and (5) can be also understood as follows. In our mechanism, the Nernst and Hall effects are caused by the asymmetric (or skew) scattering processes of quasiparticles due to CSF. Contributions from such asymmetric scattering processes to off-diagonal components of transport tensors, e.g., $\alpha_{x y}, \sigma_{x y}$, spin Hall coefficient, and so on, are proportional to $\tau^{2} / \tau_{\text {skew }}$, where $\tau$ is the scattering time due to whole scattering processes and $\tau_{\text {skew }}$ is that due to asymmetric scattering processes. This relationship can be derived phenomenologically by using the Boltzmann equation $[14,42]$. In our case, the scattering kernels which cause the skew scattering are not impurities but CSF. Therefore, $\tau_{\text {skew }}$ is independent of the purity of the system, and then $\alpha_{x y}, \sigma_{x y} \propto \tau^{2}$, in contrast to the AHE raised by impurity skew scattering, for which $\tau_{\text {skew }} \propto \tau$.

Now, we discuss to what extent our results depend on the functional form of $W\left(\boldsymbol{k}, \omega_{j}\right)$ (see Appendix G). We confirmed that the $\tau$ dependence of Eqs. (4) and (5) in the clean limit is not changed by the specific form of $W\left(\boldsymbol{k}, \omega_{j}\right)$. However, the magnitude of the transport coefficients depends on it: the contributions are decreased as the momentum-dependence of the interaction is stronger.

Here, we comment on a possible relation between our mechanism and the Berry phase (see Appendix H). The Berry curvature of chiral superconductors is proportional to the square of the superconducting gap amplitude $\Delta^{2}$ for small $|\Delta|$, and hence, the Gaussian superconducting fluctuation above $T_{c}$ is related to the Berry phase fluctuation. Also, our scenario is applicable to the Rashba $s$-wave superconductors, where our mechanism is raised by nonchiral s-wave superconducting fluctuations, which induce effective CSF associated with the Berry curvature. These observations suggest that the Berry phase fluctuation may play an important role in our mechanism.

\section{IMPLICATIONS FOR EXPERIMENTS}

We discuss the implication of our results for experiments. The Nernst effect is observed by measuring the Nernst coefficient $v^{\mathrm{NE}}$. Since the longitudinal conductivity is dominated by the Fermi liquid contribution, $\sigma_{x x}^{n}$, and also, for $\mathrm{URu}_{2} \mathrm{Si}_{2}$, $\alpha_{x y} / \sigma_{x x}^{n} \gg S \tan \Theta_{H}$, where $S$ is the Seebeck constant and $\Theta_{H}$ is the Hall angle [43], $v^{\mathrm{NE}} \approx v^{\mathrm{NE} n}+v^{\mathrm{NEFluc}}$, where $v^{\mathrm{NE} n}$ is the Fermi liquid contribution, and $\nu^{\text {NEFluc }}=\alpha_{x y}^{\text {Fluc }} / \sigma_{x x}^{n} H$ with $\alpha_{x y}^{\text {Fluc }}$ the superconducting fluctuation term. As mentioned above, $\alpha_{x y}$ due to conventional fluctuation mechanism does not depend on $\tau$, and thus $v^{\text {NEFluc }} \propto \tau^{-1}$ for nonchiral superconductors, which implies that this effect is suppressed for cleaner samples with larger $\tau$ [44]. In contrast, the CSF mechanism gives $v_{\text {chiral }}^{\text {NEFluc }} \propto \tau^{1}$ and, therefore, it is more enhanced for cleaner samples. Recently, the measurement of the Nernst effect for clean samples of $\mathrm{URu}_{2} \mathrm{Si}_{2}$ with different values of RRRs was carried out by the Kyoto group [43]. They found that the Nernst coefficient above $T_{c}$ is strongly enhanced in cleaner samples. Therefore, our scenario provides a promising explanation for this behavior.

\section{APPLICATION TO $\mathrm{Sr}_{2} \mathrm{RuO}_{4}$}

In this paper we concentrate on the application of our scenario to $\mathrm{URu}_{2} \mathrm{Si}_{2}$. However, our theory is applicable to any chiral superconductors with electron correlation. In this section, we consider the application to $\mathrm{Sr}_{2} \mathrm{RuO}_{4}$, which is a candidate for a chiral $p+i p$ superconductor [15]. This material is a strongly correlated system with spin fluctuations [45]. Therefore, also in this material, we can expect that our mechanism works. Moreover, as shown in Appendix G, the contribution to $\alpha_{x y}$ due to this mechanism is proportional to $1 /\left(T-T_{c}\right)$ in the vicinity of $T_{c}$, where this critical behavior is the same as that due to the AL mechanism in two-dimensional superconductors [5]. However, it is noted that the Ginzburg parameter of $\mathrm{Sr}_{2} \mathrm{RuO}_{4}, G_{i}^{\mathrm{Sr}_{2} \mathrm{RuO}_{4}}$, is one order smaller than that of $\mathrm{URu}_{2} \mathrm{Si}_{2}$. In fact, we have $G_{i}^{\mathrm{Sr}_{2} \mathrm{RuO}_{4}}=\frac{7 \zeta(3)}{32 \pi^{3} k_{F} \xi} \sim 10^{-5}$, where we have used $k_{F} \sim 0.75 \AA^{-1}$ of the $\gamma$ band and $\xi \sim 660 \AA$ [15]. On the other hand, the Ginzburg parameter

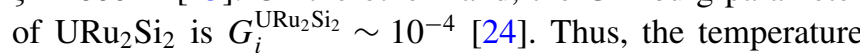
range for which fluctuation effects are prominent may be narrower for $\mathrm{Sr}_{2} \mathrm{RuO}_{4}$, compared to $\mathrm{URu}_{2} \mathrm{Si}_{2}$. Furthermore, the magnitudes of $\alpha_{x y}$ and $\sigma_{x y}$ due to the chirality fluctuation depend on the strength of electron correlation expressed by $W\left(\boldsymbol{k}, \omega_{j}\right)$, and electron correlation effects in $\mathrm{Sr}_{2} \mathrm{RuO}_{4}$ may be weaker than that of the $f$-electron-based heavy-fermion system $\mathrm{URu}_{2} \mathrm{Si}_{2}$, in which effective mass enhancement measured from the specific-heat coefficient is much larger. Thus, the experimental detection of the ANE and AHE of our scenario for $\mathrm{Sr}_{2} \mathrm{RuO}_{4}$ may be more difficult than the case of $\mathrm{URu}_{2} \mathrm{Si}_{2}$.

\section{QUANTITATIVE ESTIMATION OF THE CSF-INDUCED PARAMAGNETISM}

In this section, we comment on the possibility of the observation of the CSF-induced paramagnetism, $\chi_{\text {chiral }}$. We focus on $\mathrm{UR}_{2} \mathrm{Si}_{2}$ and $\mathrm{Sr}_{2} \mathrm{RuO}_{4}$. For these chiral superconductors, the ratios of $\chi_{\text {chiral }}$ to $\chi_{\text {dia }}$ are, respectively, given by

$$
\begin{aligned}
& \left|\frac{\chi_{\mathrm{chiral}}^{\mathrm{URu}_{2} \mathrm{Si}_{2}}}{\chi_{\mathrm{dia}}^{3 D}}\right| \sim \frac{4}{\left(k_{F} \xi\right)^{3}} \frac{T_{c}}{k_{F} v_{F}} \frac{1}{[N(0) g]^{2}}<10^{-3}, \\
& \left|\frac{\chi_{\mathrm{chiral}}^{\mathrm{Sr}_{2} \mathrm{RuO}_{4}}}{\chi_{\mathrm{dia}}^{2 D}}\right| \sim \frac{1}{\left(k_{F} \xi_{a b}\right)^{4}[N(0) g]^{2}}<10^{-8} .
\end{aligned}
$$

To derive these relations, we used the expressions for fluctuation-induced diamagnetism, $\chi_{\text {dia }}^{3 D} \sim-e^{2} v_{F} /\left(4 \pi^{2} \varepsilon^{1 / 2}\right)$ and $\chi_{\text {dia }}^{2 D} \sim-e^{2} T \xi_{a b}^{2} / \varepsilon a_{c}$ [30], and that for the paramagnetism for the two-dimensional chiral $p_{x} \pm i p_{y}$ superconductor: $\chi_{\text {chiral }}^{\mathrm{Sr}_{2} \mathrm{RuO}_{4}} \sim e^{2} T /\left\{k_{F}^{4} \xi_{a b}^{2}[N(0) g]^{2} \varepsilon a_{c}\right\}$, which can be obtain by calculations similar to that for derivation of Eq. (6). Here $\xi_{a b}$ is the $a b$-plane coherence length and $a_{c}$ is the interlayer spacing. We also used the material parameters, $k_{F}, v_{F}, \xi, \xi_{a b}$, and $T_{c}$, of $\mathrm{URu}_{2} \mathrm{Si}_{2}$ and $\mathrm{Sr}_{2} \mathrm{RuO}_{4}[15,46]$, and assumed that the value of $N(0) g$ is larger than that of typical weak-coupling BCS-type superconductors: $N(0) g>0.1$ [47].

As seen from the above estimations, in both materials, it is difficult to detect the divergent paramagnetism, $\chi_{\text {chiral }}$, 
because it is overwhelmed by the diamagnetism with the same critical behavior. However, as seen in Eq. (7), we expect that, in other chiral superconductors with shorter coherence length and smaller Fermi energy, this magnetism can be observed.

\section{SUMMARY}

We elucidate the mechanism of the anomalous Nernst and Hall effects raised not by the Lorentz force, but by asymmetric scatterings due to CSF above $T_{c}$ in chiral superconductors. These effects can be gigantic for cleaner samples, which makes sharp contrast to conventional mechanisms of Gaussianfluctuation-induced transverse transport phenomena. We propose that our theory can be promisingly tested for $\mathrm{URu}_{2} \mathrm{Si}_{2}$, which is believed to be a chiral $d+i d$ superconductor with strong superconducting fluctuations near and above $T_{c}$.

\section{ACKNOWLEDGMENTS}

We thank T. Yamashita, S. Tonegawa, Y. Tsuruhara, Y. Shimoyama, T. Shibauchi, and Y. Matsuda for providing experimental data which gave us motivation of this study, and A. Shitade, K. Shiozaki, T. Nomoto, and R. Ikeda for helpful discussions. This work was supported by Grants-inAid for Scientific Research from MEXT of Japan [Grants No. 23540406, No. 25220711, and No. 25103714 (KAKENHI on Innovative Areas Topological Quantum Phenomena)]. H.S. is supported by a JSPS Fellowship for Young Scientists.

\section{APPENDIX A: BPS UNDER MAGNETIC FIELD}

In this section, we derive the expression of the BPS, Eq. (3) in the main text, under a homogeneous magnetic field $(0,0, H)$. The interaction term of the Hamiltonian (1) for the chirality $C=+1$ channel can be rewritten in real-space representation as

$$
\begin{aligned}
H^{\mathrm{int}} & =-g \int d \boldsymbol{r}\left[V^{+}\left(\frac{-i\left(\partial_{1}-\partial_{2}\right)}{2}, \frac{-i\left(\partial_{1}^{\prime}-\partial_{2}^{\prime}\right)}{2}\right) c_{\uparrow}^{*}\left(\boldsymbol{r}_{1}\right) c_{\downarrow}^{*}\left(\boldsymbol{r}_{2}\right) c_{\downarrow}\left(\boldsymbol{r}_{2}^{\prime}\right) c_{\uparrow}\left(\boldsymbol{r}_{1}^{\prime}\right)\right]_{r_{1}, \boldsymbol{r}_{2}, \boldsymbol{r}_{1}^{\prime}, \boldsymbol{r}_{2}^{\prime} \rightarrow \boldsymbol{r}} \\
& =-g \int d \boldsymbol{r}\left[\phi\left(\frac{-i\left(\partial_{1}-\partial_{2}\right)}{2}\right) \phi^{\dagger}\left(\frac{-i\left(\partial_{1}^{\prime}-\partial_{2}^{\prime}\right)}{2}\right) c_{\uparrow}^{*}\left(\boldsymbol{r}_{1}\right) c_{\downarrow}^{*}\left(\boldsymbol{r}_{2}\right) c_{\downarrow}\left(\boldsymbol{r}_{2}^{\prime}\right) c_{\uparrow}\left(\boldsymbol{r}_{1}^{\prime}{ }_{1}\right)\right]_{r_{1}, \boldsymbol{r}_{2}, \boldsymbol{r}_{1}^{\prime}, \boldsymbol{r}_{2}^{\prime} \rightarrow \boldsymbol{r}} .
\end{aligned}
$$

Therefore, the BPS of this channel is given by

$$
\tilde{\Pi}_{C=1}\left(\boldsymbol{x}, \boldsymbol{y}, \omega_{l} ; H\right)=\left.T \sum_{\varepsilon_{n}}\left[\phi\left(\frac{-i\left(\partial_{1}-\partial_{2}\right)}{2}\right) \phi^{\dagger}\left(\frac{-i\left(\partial_{1}^{\prime}-\partial_{2}^{\prime}\right)}{2}\right) \tilde{G}\left(\boldsymbol{r}_{1}^{\prime}, \boldsymbol{r}_{1}, \varepsilon_{n+l} ; H\right) \tilde{G}\left(\boldsymbol{r}_{2}^{\prime}, \boldsymbol{r}_{2},-\varepsilon_{n} ; H\right)\right]\right|_{\substack{\boldsymbol{r}_{1}^{\prime}, \boldsymbol{r}_{2}^{\prime} \rightarrow \boldsymbol{x}, \boldsymbol{r}, \boldsymbol{r}_{2} \rightarrow \boldsymbol{y} \\ \boldsymbol{y}_{1}}} .
$$

In the presence of a uniform magnetic field, the one-particle Green's function is $\tilde{G}\left(\boldsymbol{r}^{\prime}, \boldsymbol{r}, \varepsilon_{n} ; H\right)=e^{-i e \Phi\left(\boldsymbol{r}^{\prime}, \boldsymbol{r}\right)} G_{\mathrm{core}}\left(\boldsymbol{r}^{\prime}-\boldsymbol{r}, \varepsilon_{n} ; H\right)$, where $\Phi(\boldsymbol{x}, \boldsymbol{y})=\int_{\boldsymbol{x}}^{\boldsymbol{y}} \boldsymbol{A}(\boldsymbol{r}) d \boldsymbol{r}$ is an integral of the vector potential $\boldsymbol{A}(\boldsymbol{r})$ along a straight line, and $G_{\text {core }}\left(\boldsymbol{r}^{\prime}-\boldsymbol{r}, \varepsilon_{n} ; H\right)$ is the "core" Green's function, which is translation (then it is a function of $\boldsymbol{r}^{\prime}-\boldsymbol{r}$ ), $c$-axis rotation, and gauge invariant [31-33], and is given as the solution of

$$
\left[i \varepsilon_{n}-\frac{1}{2 m}\left(-i \nabla_{\rho}+\frac{e}{2} \boldsymbol{\rho} \times \boldsymbol{H}\right)^{2}+\mu-\hat{\Sigma}\right] G_{\mathrm{core}}\left(\boldsymbol{\rho}, \varepsilon_{n} ; H\right)=\delta(\boldsymbol{\rho})
$$

where $\rho=\boldsymbol{r}^{\prime}-\boldsymbol{r}$, and $\hat{\Sigma}$ is the self-energy that also has the same symmetries as mentioned above.

To proceed further, we take the Landau gauge, $\boldsymbol{A}(\boldsymbol{r})=(0, x H, 0)$, although the final results (A4)-(A6) are correct for any gauge choice as shown in the last part of this section. For this gauge, $\Phi\left(\boldsymbol{r}^{\prime}, \boldsymbol{r}\right)=\frac{e H}{2}\left(x^{\prime}+x\right)\left(y-y^{\prime}\right)$. Now, we introduce a $p_{z}$-wave structure factor $\phi^{z}(\boldsymbol{k})=\sqrt{3} k_{z} / k_{F}$, and let $\theta=-i e\left[\Phi\left(\boldsymbol{r}_{1}^{\prime}, \boldsymbol{r}_{1}\right)+\Phi\left(\boldsymbol{r}_{2}^{\prime}, \boldsymbol{r}_{2}\right)\right], \phi_{12}=\phi\left(\frac{-i\left(\partial_{1}-\partial_{2}\right)}{2}\right)$, and $\phi_{12}^{z}=$ $\phi^{z}\left(\frac{-i\left(\partial_{1}-\partial_{2}\right)}{2}\right)$. The commutators of $\phi$ and $\theta$ are given by $\left[\phi_{12}, \theta\right]=\frac{1}{4} \sqrt{\frac{5}{2}} \frac{i e H}{k_{F}}\left(-x_{1}-x_{1}^{\prime}+x_{2}+x_{2}^{\prime}+i y_{1}-i y_{1}^{\prime}-i y_{2}^{\prime}+i y_{2}^{\prime}\right) \phi_{12}^{z}$, $\left[\phi_{1^{\prime} 2^{\prime}}^{\dagger}, \theta\right]=\frac{1}{4} \sqrt{\frac{5}{2}} \frac{i e H}{k_{F}}\left(-x_{1}-x_{1}^{\prime}+x_{2}+x_{2}^{\prime}+i y_{1}-i y_{1}^{\prime}-i y_{2}^{\prime}+i y_{2}^{\prime}\right) \phi_{1^{\prime} 2^{\prime}}^{z}$, and $\left[\phi_{12},\left[\phi_{1^{\prime} 2^{\prime}}^{\dagger} \theta\right]\right]=-\frac{5 e H}{4 k_{F}^{2}} \phi_{12}^{z} \phi_{1^{\prime} 2^{\prime}}^{z}$. Then, [ $\left.\phi_{12}, \theta\right] \rightarrow 0$ and $\left[\phi_{1^{\prime} 2^{\prime}}^{\dagger}, \theta\right] \rightarrow 0$ as $\boldsymbol{r}_{1}^{\prime}, \boldsymbol{r}_{2}^{\prime} \rightarrow \boldsymbol{x}$ and $\boldsymbol{r}_{1}, \boldsymbol{r}_{2} \rightarrow \boldsymbol{y}$. By using these commutation relations and the formulas $e^{B} A e^{-B}=$ $A+[B, A]+[B,[B, A]]+\cdots$ and $[P Q, R]=P[Q, R]+[P, R] Q$, we obtain

$$
\begin{aligned}
\tilde{\Pi}_{C=1}\left(\boldsymbol{x}, \boldsymbol{y}, \omega_{q} ; H\right)= & e^{-2 i e \Phi(\boldsymbol{x}, \boldsymbol{y})}\left[T \sum_{\varepsilon_{n}} \phi_{12} \phi_{1^{\prime} 2^{\prime}}^{\dagger} G_{\mathrm{core}}\left(\boldsymbol{r}_{1}^{\prime}-\boldsymbol{r}_{1}, \varepsilon_{n+q} ; H\right) G_{\mathrm{core}}\left(\boldsymbol{r}_{2}^{\prime}-\boldsymbol{r}_{2},-\varepsilon_{n} ; H\right)\right. \\
& \left.-\frac{5 e H}{4 k_{F}^{2}} T \sum_{\varepsilon_{n}} \phi_{12}^{z} \phi_{1^{\prime} 2^{\prime}}^{z} G_{\mathrm{core}}\left(\boldsymbol{r}_{1}^{\prime}-\boldsymbol{r}_{1}, \varepsilon_{n+q} ; H\right) G_{\mathrm{core}}\left(\boldsymbol{r}_{2}^{\prime}-\boldsymbol{r}_{2},-\varepsilon_{n} ; H\right)\right] \underset{\substack{\boldsymbol{r}_{1}^{\prime}, \boldsymbol{r}_{2}^{\prime} \rightarrow \boldsymbol{x}, \boldsymbol{r}_{1}, \boldsymbol{r}_{2} \rightarrow \boldsymbol{y}}}{=} \\
= & e^{-i 2 e \Phi(\boldsymbol{x}, \boldsymbol{y})}\left[\Pi\left(\boldsymbol{x}-\boldsymbol{y}, \omega_{q} ; H\right)-\frac{5 e H}{4 k_{F}^{2}} \Pi^{\prime}\left(\boldsymbol{x}-\boldsymbol{y}, \omega_{q} ; H\right)\right]
\end{aligned}
$$


where

$$
\begin{aligned}
& \Pi\left(\boldsymbol{\rho}, \omega_{q} ; H\right)=\left[T \sum_{\varepsilon_{n}}|\phi|^{2}\left(\frac{-i\left(\partial_{\boldsymbol{\rho}_{1}}-\partial_{\boldsymbol{\rho}_{2}}\right)}{2}\right) G_{\text {core }}\left(\boldsymbol{\rho}_{1}, \varepsilon_{n+q} ; H\right) G_{\text {core }}\left(\boldsymbol{\rho}_{2},-\varepsilon_{n} ; H\right)\right]_{\boldsymbol{\rho}_{1}, \boldsymbol{\rho}_{2} \rightarrow \boldsymbol{\rho}}, \\
& \Pi^{\prime}\left(\boldsymbol{\rho}, \omega_{q} ; H\right)=\left[T \sum_{\varepsilon_{n}}\left|\phi^{z}\right|^{2}\left(\frac{-i\left(\partial_{\boldsymbol{\rho}_{1}}-\partial_{\boldsymbol{\rho}_{2}}\right)}{2}\right) G_{\mathrm{core}}\left(\boldsymbol{\rho}_{1}, \varepsilon_{n+q} ; H\right) G_{\mathrm{core}}\left(\boldsymbol{\rho}_{2},-\varepsilon_{n} ; H\right)\right]_{\boldsymbol{\rho}_{1}, \boldsymbol{\rho}_{2} \rightarrow \boldsymbol{\rho}}
\end{aligned}
$$

are "core" BPSs, which preserve spatial translation, $c$-axis rotation, and gauge invariances. Here $|\phi(\boldsymbol{k})|^{2}=15 k_{z}^{2}\left(k_{x}^{2}+\right.$ $\left.k_{y}^{2}\right) / 2 k_{F}^{4}$ and $\left|\phi^{z}(\boldsymbol{k})\right|^{2}=3 k_{z}^{2} / k_{F}^{2}$. Then, we arrive at Eq. (3) for $C=+1$. Carrying out similar calculations with the effective interaction term $V^{-}$, we can obtain Eq. (3) for $C=-1$.

So far we have used the Landau gauge. However, by using the formulas, $\left.\left[\phi_{12}, e^{-i\left[\chi\left(\boldsymbol{r}_{1}\right)+\chi\left(\boldsymbol{r}_{2}\right)\right]}\right]\right|_{\boldsymbol{r}_{1}, \boldsymbol{r}_{2} \rightarrow \boldsymbol{y}}=0$, etc., with $\chi$ an arbitrary function, we can prove that the final results, (A4)(A6), are gauge invariant.

\section{APPENDIX B: NERNST AND HALL CONDUCTIVITIES}

In this section, we present the derivation of the Nernst and Hall conductivities for the ANE and AHE caused by the chiral superconducting fluctuation mechanism, Eqs. (4) and (5) in the main text. Formally, to obtain the whole contributions from the superconducting fluctuations to the Kubo term of the Nernst conductivity and the Hall conductivity, one has to evaluates all possible Feynman diagrams that consist of two current vertices, superconducting fluctuation propagators, $\tilde{L}_{C}$, and Green's functions, $\tilde{G}$, in a magnetic field. We focus on the case with a weak magnetic field, where the Nernst and Hall conductivities are linear in $H$. Up to the linear order in $H$, the fluctuation propagator (2) is divided into two parts:

$$
\tilde{L}_{C}=\tilde{L}_{0}+\tilde{L}_{C}^{\prime}+O\left(H^{2}\right),
$$

where

$$
\begin{aligned}
& \tilde{L}_{0}^{-1}\left(\boldsymbol{x}, \boldsymbol{y}, \omega_{q} ; H\right) \\
& \quad=-\frac{\delta(\boldsymbol{x}-\boldsymbol{y})}{g}+e^{-i 2 e \Phi(\boldsymbol{x}, \boldsymbol{y})} \Pi\left(\boldsymbol{x}-\boldsymbol{y}, \omega_{q} ; H\right)
\end{aligned}
$$

is the conventional part of the fluctuation propagator, and

$$
\tilde{L}_{C}^{\prime}\left(\boldsymbol{x}-\boldsymbol{y}, \omega_{q} ; H\right)=C\left(\frac{5 e H}{4 k_{F}^{2} g}\right)\left[L\left(\boldsymbol{x}-\boldsymbol{y}, \omega_{q}\right)\right]^{2}
$$

is the chirality-dependent one which is characteristic of chiral superconductors. Here $L\left(\boldsymbol{x}-\boldsymbol{y}, \omega_{q}\right)$ is the fluctuation propagator of the $d_{z x} \pm i d_{z y}$-wave channel in zero magnetic field. The concrete expression of $L\left(\boldsymbol{x}-\boldsymbol{y}, \omega_{q}\right)$ and its derivation are given in Appendix C. From Eq. (B1), we see that in the linear order of $H$, the whole contributions to the Nernst and Hall conductivities are separated into two parts: (A) the contribution from diagrams which do not include the chirality-dependent fluctuation propagator, $\tilde{L}_{C}^{\prime}$, and (B) the contribution from diagrams which include one chirality-dependent fluctuation propagator $\tilde{L}_{C}^{\prime}$.

The contribution (A) also appears for the case of nonchiral superconductors, such as $s$ - and $d_{x^{2}-y^{2}}$-wave pairings, which can be described by conventional theories $[30,38,39]$, and therefore, physically, this part corresponds to the contributions due to the Lorentz force on quasiparticles and fluctuating Cooper pairs. On the other hand, (B) is unique to chiral superconductors, raised by chirality polarization, and as shown below, associated with the ANE and AHE caused by the chiral superconducting fluctuation mechanism without the Lorentz force.

From now on, we concentrate on the latter contribution, and write down the correlation functions for the Nernst and Hall conductivities in the form

$$
A_{x y}\left(\omega_{l}\right)=-2 e T \sum_{\boldsymbol{q}, \omega_{q}, C= \pm 1} \tilde{L}_{C}^{\prime}\left(\boldsymbol{q}, \omega_{q} ; H\right) \bar{A}_{C}\left(\boldsymbol{q}, \omega_{q} ; \omega_{l}\right),
$$

where only the odd part of $\bar{A}_{C}$ with respect to time-reversal operation, $C \rightarrow-C$, gives nonzero contributions, since $\tilde{L}_{C}^{\prime}$ is odd.

We examine the leading-order diagrams belonging to (B), which give the dominant contribution. It is found that the three diagrams which give leading-order contributions in conventional theories, i.e., the Aslamazov-Larkin (AL), MakiThompson (MT), and density-of-states (DOS) diagrams (upper panel in Fig. 1), do not contribute in this case, and generally, all contributions from diagrams belonging to the classes of the lower panel in Fig. 1 are zero. The reason is that in these diagrams the paring functions appear as $|\phi|^{2}$, and, therefore, their contributions to $\bar{A}_{C}$ have only an even part with respect

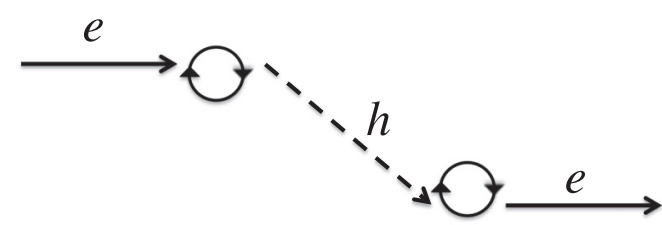

FIG. 4. Schematic picture of a scattering process raised only by chiral superconducting fluctuations. The solid and dashed arrows represent a propagating electron and hole, respectively, and the circle with arrows means the short-lived Cooper pairs with the chirality $C=$ +1 . By one skew-scattering event, an electron is transformed into a hole (i.e., so-called Andreev reflection). Thus, processes with an even number of the events do not change the momentum of the electron and therefore do not contribute to the Nernst and Hall effects. Also, processes with an odd number of events are suppressed after averaging over fluctuations owing to the conservation of the electron number in the normal state (this figure is one example of the even cases). Thus, to obtain the ANE and AHE due to skew scattering, we need additional scattering processes which disturb the above-mentioned cancellation of skew scatterings. 
to time reversal:

$$
\bar{A}_{C}^{\mathrm{AL}, \mathrm{MT}, \mathrm{DOS}}=\bar{A}_{-C}^{\mathrm{AL}, \mathrm{MT}, \mathrm{DOS}},
$$

which do not contribute to the correlation function as mentioned above. This means that the information of the chirality disappears in these diagrams. This is physically understood as follows. Although the chiral superconducting fluctuation raises skew scattering of electrons, electrons are scattered into holes by the superconducting fluctuation, and vice versa, and also holes are skew scattered in the direction opposite to electron skew scattering. As a result, the secondorder processes of scattering due to the chirality fluctuation lead to the cancellation of skew scattering. We depict this cancellation schematically in Fig. 4. On the other hand, if there are additional scattering processes with momentum transfer which occur between two skew-scattering events, the cancellation becomes incomplete, and we have finite contributions from skew scattering to transverse transport coefficients. The lowest order diagrams which give nonzero contributions in this manner are depicted in Fig. 2 in the main text. For clarity, we depict them more explicitly in Figs. 5(a)-5(c). In these diagrams, a renormalized four-point vertex, $W\left(\boldsymbol{k}, \omega_{j}\right)$ (double line), raised by electron-electron interaction is inserted. To proceed further, we introduce a simple model: $W\left(\boldsymbol{k}, \omega_{j}\right)=W_{0} /\left(1+\left|\omega_{j}\right| / \Gamma\right)$, the four-point vertex mediated via the short-range spin fluctuation. We use this toy model for $W\left(\boldsymbol{k}, \omega_{j}\right)$ to carry out calculations explicitly. However, we would like to stress that the important features of the main results for the Nernst and Hall conductivities obtained below are qualitatively not changed, even if we use more realistic momentum-dependent $W\left(\boldsymbol{k}, \omega_{j}\right)$. We discuss this point in Appendix G.

Now, we calculate contributions from diagrams (a), (b), and (c) and their mirror images in Fig. 2 to the Kubo terms of the Nernst conductivity, $\alpha_{x y}^{(i) \text { Kubo }}(i=a, b$, and $c$ ), and the Hall conductivity, $\sigma_{x y}^{(i)}(i=a, b$, and $c)$. They are, respectively, given by

$$
\begin{aligned}
\alpha_{x y}^{(i) \text { Kubo }} & =\left.\frac{1}{T} \frac{A_{\alpha \beta}^{(i) R}(\omega)}{(-i \omega)}\right|_{\omega \rightarrow 0}, \\
\sigma_{x y}^{(i)} & =\left.\frac{S_{\alpha \beta}^{(i) R}(\omega)}{(-i \omega)}\right|_{\omega \rightarrow 0},
\end{aligned}
$$

where $A_{\alpha \beta}^{(i) R}(\omega)$ is the retarded heat current-charge current correlation function, and $S_{\alpha \beta}^{(i) R}(\omega)$ is the retarded charge current-charge current correlation function. Their correspond-
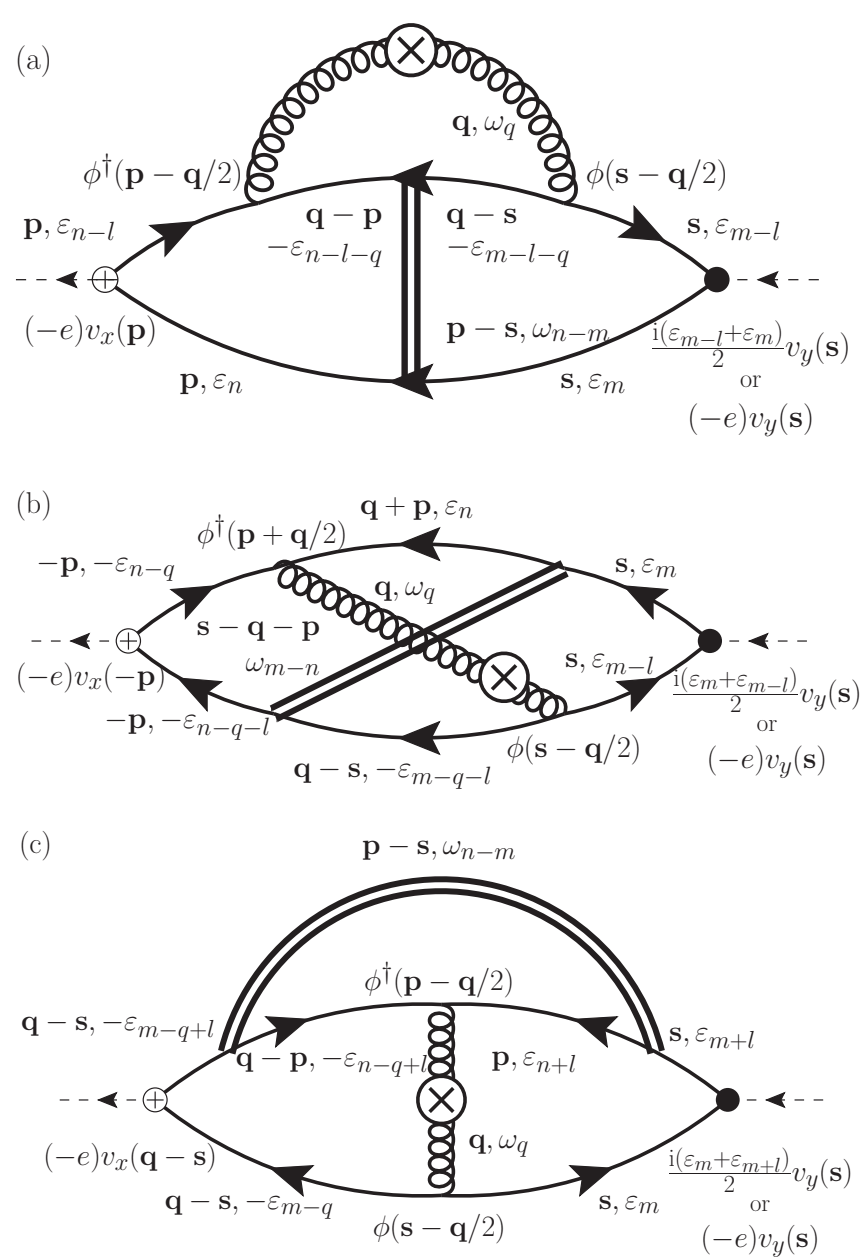

FIG. 5. Diagrams (a), (b), and (c) of Fig. 2 with wave numbers and frequencies explicitly shown.

ing correlation functions with the Matsubara frequencies are, respectively,

$$
A_{x y}^{(i)}\left(\omega_{l}\right)=-2 e T \sum_{\omega_{q}, \boldsymbol{q}, C= \pm 1} \tilde{L}_{C}^{\prime}\left(\boldsymbol{q}, \omega_{q} ; H\right) \bar{A}_{C}^{(i)}\left(\boldsymbol{q}, \omega_{q} ; \omega_{l}\right),
$$

$$
S_{x y}^{(i)}\left(\omega_{l}\right)=2 e^{2} T \sum_{\omega_{q}, \boldsymbol{q}, C= \pm 1} \tilde{L}_{C}^{\prime}\left(\boldsymbol{q}, \omega_{q} ; H\right) \bar{S}_{C}^{(i)}\left(\boldsymbol{q}, \omega_{q} ; \omega_{l}\right),
$$

where $\omega_{q}$ and $\omega_{l}$ are the Matsubara frequencies, and $\boldsymbol{q}$ is a wave number. Here,

$$
\begin{aligned}
& \bar{A}_{C=1}^{(i)}\left(\boldsymbol{q}, \omega_{q} ; \omega_{l}\right)=-2 T^{2} \operatorname{Re}\left[\sum_{n, m} X^{(i)}\left(\boldsymbol{q}, n, \omega_{q} ; \omega_{l}\right) Y^{(i)}\left(\boldsymbol{q}, m, \omega_{q} ; \omega_{l}\right) \frac{W_{0}}{1+\left|\omega_{n-m}\right| / \Gamma}\right], \\
& \bar{S}_{C=1}^{(i)}\left(\boldsymbol{q}, \omega_{q} ; \omega_{l}\right)=-2 T^{2} \operatorname{Re}\left[\sum_{n, m} X^{(i)}\left(\boldsymbol{q}, n, \omega_{q} ; \omega_{l}\right) Z^{(i)}\left(\boldsymbol{q}, m, \omega_{q} ; \omega_{l}\right) \frac{W_{0}}{1+\left|\omega_{n-m}\right| / \Gamma}\right],
\end{aligned}
$$

and

$$
X^{(a)}\left(\boldsymbol{q}, n, \omega_{q} ; \omega_{l}\right)=\sum_{\boldsymbol{p}} \phi^{\dagger}\left(\boldsymbol{p}-\frac{\boldsymbol{q}}{2}\right) G\left(\boldsymbol{q}-\boldsymbol{p},-\varepsilon_{n-l-q}\right) G\left(\boldsymbol{p}, \varepsilon_{n-l}\right) G\left(\boldsymbol{p}, \varepsilon_{n}\right) v_{x}(\boldsymbol{p})
$$




$$
\begin{gathered}
Y^{(a)}\left(\boldsymbol{q}, m, \omega_{q} ; \omega_{l}\right)=\sum_{s} \phi\left(s-\frac{\boldsymbol{q}}{2}\right) G\left(\boldsymbol{q}-\boldsymbol{s},-\varepsilon_{m-l-q}\right) G\left(\boldsymbol{s}, \varepsilon_{m-l}\right) G\left(\boldsymbol{s}, \varepsilon_{m}\right) \frac{i\left(\varepsilon_{m-l}+\varepsilon_{m}\right)}{2} v_{y}(\boldsymbol{s}), \\
Z^{(a)}\left(\boldsymbol{q}, m, \omega_{q} ; \omega_{l}\right)=\sum_{\boldsymbol{s}} \phi\left(\boldsymbol{s}-\frac{\boldsymbol{q}}{2}\right) G\left(\boldsymbol{q}-\boldsymbol{s},-\varepsilon_{m-l-q}\right) G\left(\boldsymbol{s}, \varepsilon_{m-l}\right) G\left(\boldsymbol{s}, \varepsilon_{m}\right) v_{y}(\boldsymbol{s}), \\
X^{(b)}\left(\boldsymbol{q}, n, \omega_{q} ; \omega_{l}\right)=\sum_{\boldsymbol{p}} \phi^{\dagger}\left(\boldsymbol{p}+\frac{\boldsymbol{q}}{2}\right) G\left(\boldsymbol{q}+\boldsymbol{p}, \varepsilon_{n}\right) G\left(-\boldsymbol{p},-\varepsilon_{n-q}\right) G\left(-\boldsymbol{p},-\varepsilon_{n-q-l}\right) v_{x}(-\boldsymbol{p}), \\
Y^{(b)}\left(\boldsymbol{q}, m, \omega_{q} ; \omega_{l}\right)=\sum_{\boldsymbol{s}} \phi\left(\boldsymbol{s}-\frac{\boldsymbol{q}}{2}\right) G\left(\boldsymbol{q}-\boldsymbol{s},-\varepsilon_{m-q-l}\right) G\left(\boldsymbol{s}, \varepsilon_{m-l}\right) G\left(\boldsymbol{s}, \varepsilon_{m}\right) \frac{i\left(\varepsilon_{m}+\varepsilon_{m-l}\right)}{2} v_{y}(\boldsymbol{s}), \\
Z^{(b)}\left(\boldsymbol{q}, m, \omega_{q} ; \omega_{l}\right)=\sum_{\boldsymbol{s}} \phi\left(\boldsymbol{s}-\frac{\boldsymbol{q}}{2}\right) G\left(\boldsymbol{q}-\boldsymbol{s},-\varepsilon_{m-q-l}\right) G\left(\boldsymbol{s}, \varepsilon_{m-l}\right) G\left(\boldsymbol{s}, \varepsilon_{m}\right) v_{y}(\boldsymbol{s}), \\
X^{(c)}\left(\boldsymbol{q}, n, \omega_{q} ; \omega_{l}\right)=\sum_{\boldsymbol{p}} \phi^{\dagger}\left(\boldsymbol{p}-\frac{\boldsymbol{q}}{2}\right) G\left(\boldsymbol{q}-\boldsymbol{p},-\varepsilon_{n+l-q}\right) G\left(\boldsymbol{p}, \varepsilon_{n+l}\right), \\
Y^{(c)}\left(\boldsymbol{q}, m, \omega_{q} ; \omega_{l}\right)=\sum_{\boldsymbol{s}} \phi\left(\boldsymbol{s}-\frac{\boldsymbol{q}}{2}\right) G\left(\boldsymbol{q}-\boldsymbol{s},-\varepsilon_{m-q}\right) G\left(\boldsymbol{s}, \varepsilon_{m}\right) G\left(\boldsymbol{s}, \varepsilon_{m+l}\right) G\left(\boldsymbol{q}-\boldsymbol{s},-\varepsilon_{m+l-q}\right) v_{x}(\boldsymbol{q}-\boldsymbol{s}) \frac{i\left(\varepsilon_{m}+\varepsilon_{m+l}\right)}{2} v_{y}(\boldsymbol{s}), \\
Z^{(c)}\left(\boldsymbol{q}, m, \omega_{q} ; \omega_{l}\right)=\sum_{\boldsymbol{s}} \phi\left(\boldsymbol{s}-\frac{\boldsymbol{q}}{2}\right) G\left(\boldsymbol{q}-\boldsymbol{s},-\varepsilon_{m-q}\right) G\left(\boldsymbol{s}, \varepsilon_{m}\right) G\left(\boldsymbol{s}, \varepsilon_{m+l}\right) G\left(\boldsymbol{q}-\boldsymbol{s},-\varepsilon_{m+l-q}\right) v_{x}(\boldsymbol{q}-\boldsymbol{s}) v_{y}(\boldsymbol{s}) . \\
(\mathrm{B} 20)
\end{gathered}
$$

We can also obtain the expressions for $\bar{A}_{C=-1}^{(i)}$ and $\bar{S}_{C=-1}^{(i)}$ by using chirality-inversion (time-reversal) transformation, $\phi \rightarrow \phi^{\dagger}$ and $\phi^{\dagger} \rightarrow \phi$ in $X^{(i)}, Y^{(i)}$, and $Z^{(i)}$, of Eqs. (B10)-(B20). In the above equations, the one-particle Green's function, chiral $d_{z x}+i d_{z y^{-}}$ wave pairing symmetry function, and velocity of quasiparticles are defined as $G\left(\boldsymbol{k}, \varepsilon_{k}\right)^{-1}=i \tilde{\varepsilon}_{k}-\xi_{\boldsymbol{k}}=i\left[\varepsilon_{k}+\operatorname{sgn}\left(\varepsilon_{\mathrm{k}}\right) / 2 \tau\right]-\xi_{\boldsymbol{k}}$, $\phi(\boldsymbol{k})=\sqrt{15 / 2} k_{z}\left(k_{x}+i k_{y}\right) / k_{F}^{2}$, and $\boldsymbol{v}(\boldsymbol{k})=\partial \xi_{\boldsymbol{k}} / \partial \boldsymbol{k}$, respectively. Here $\tau$ is the relaxation time of quasiparticles. For simplicity, we take the spherical Fermi surface: $\xi_{k}=k^{2} / 2 m-\mu$, where $m$ and $\mu$ are the mass and chemical potential of the quasiparticles, respectively.

We henceforth neglect quantum superconducting fluctuations keeping only terms with $\omega_{q}=0$. Since singular contributions at $T_{c}$ come from long-wavelength regions where the center-of-mass momentum of fluctuating Cooper pairs $q$ is small, we concentrate on the analysis of $\tilde{A}^{(i)}$ and $\tilde{S}^{(i)}$ for small $q$. Then, expanding $X^{(i)}, Y^{(i)}$, and $Z^{(i)}$ as power series of $q / k_{F}$, we obtain

$$
\begin{aligned}
& X^{(a)}\left(\boldsymbol{q}, n, \omega_{q}=0 ; \omega_{l}\right) \\
& =N(0) q_{z}\left[i \pi \sqrt{\frac{5}{24}} \frac{v_{F}}{k_{F}} \frac{\operatorname{sgn}(n+1 / 2)}{\left(\left|\tilde{\varepsilon}_{n}\right|+\left|\tilde{\varepsilon}_{n-l}\right|\right)\left|\tilde{\varepsilon}_{n-l}\right|}-\frac{\pi}{2} \sqrt{\frac{1}{30}} v_{F}^{2}\left\{\begin{array}{ll}
\frac{\left|\tilde{\varepsilon}_{n}\right|+3\left|\tilde{\varepsilon}_{n-l}\right|}{\left|\tilde{\varepsilon}_{n-l}\right|^{2}\left(\left|\tilde{\varepsilon}_{n}\right|+\left|\tilde{\varepsilon}_{n-l}\right|\right)^{2}}, & \text { for } \quad(n-l+1 / 2)(n+1 / 2)>0 \\
\frac{-1}{\left|\tilde{\varepsilon}_{n-l}\right|^{2}\left(\left|\tilde{\varepsilon}_{n}\right|+\left|\tilde{\varepsilon}_{n-l}\right|\right)}, & \text { for } \quad(n-l+1 / 2)(n+1 / 2)<0
\end{array}\right]\right. \\
& +O\left(\left(\frac{q}{k_{F}}\right)^{2}\right) \\
& Y^{(a)}\left(\boldsymbol{q}, m, \omega_{q}=0 ; \omega_{l}\right)=-\frac{\left(\varepsilon_{m-l}+\varepsilon_{m}\right)}{2} X^{(a)}\left(q, m, \omega_{q}=0 ; \omega_{l}\right), \\
& Z^{(a)}\left(\boldsymbol{q}, m, \omega_{q}=0 ; \omega_{l}\right)=i X^{(a)}\left(q, m, \omega_{q}=0 ; \omega_{l}\right),
\end{aligned}
$$

$$
\begin{aligned}
& X^{(b)}\left(\boldsymbol{q}, n, \omega_{q}=0 ; \omega_{l}\right) \\
& =N(0) q_{z}\left[-i \pi \sqrt{\frac{5}{24}} \frac{v_{F}}{k_{F}} \frac{\operatorname{sgn}(n-l+1 / 2)}{\left(\left|\tilde{\varepsilon}_{n}\right|+\left|\tilde{\varepsilon}_{n-l}\right|\right)\left|\tilde{\varepsilon}_{n}\right|}-\frac{\pi}{2} \sqrt{\frac{1}{30}} v_{F}^{2}\left\{\begin{array}{ll}
\frac{\left|\tilde{\varepsilon}_{n-l}\right|+3\left|\tilde{\varepsilon}_{n}\right|}{\left|\tilde{\varepsilon}_{n}\right|^{2}\left(\left|\tilde{\varepsilon}_{n-l}\right|+\left|\tilde{\varepsilon}_{n}\right|\right)^{2}}, & \text { for } \quad(n-l+1 / 2)(n+1 / 2)>0 \\
\frac{-1}{\left|\tilde{\varepsilon}_{n}\right|^{2}\left(\left|\tilde{\varepsilon}_{n-l}\right|+\left|\tilde{\varepsilon}_{n}\right|\right)}, & \text { for } \quad(n-l+1 / 2)(n+1 / 2)<0
\end{array}\right\}\right] \\
& +O\left(\left(\frac{q}{k_{F}}\right)^{2}\right)
\end{aligned}
$$




$$
\begin{aligned}
& Y^{(b)}\left(\boldsymbol{q}, m, \omega_{q}=0 ; \omega_{l}\right) \\
& =N(0) q_{z} \frac{\varepsilon_{m}+\varepsilon_{m-l}}{2}\left[-i \pi \sqrt{\frac{5}{24}} \frac{v_{F}}{k_{F}} \frac{\operatorname{sgn}(m+1 / 2)}{\left(\left|\tilde{\varepsilon}_{m}\right|+\left|\tilde{\varepsilon}_{m-l}\right|\right)\left|\tilde{\varepsilon}_{m-l}\right|}\right. \\
& \left.+\frac{\pi}{2} \sqrt{\frac{1}{30}} v_{F}^{2}\left\{\begin{array}{lll}
\frac{\left|\tilde{\varepsilon}_{m}\right|+3\left|\tilde{\varepsilon}_{m-l}\right|}{\left|\tilde{\varepsilon}_{m-l}\right|^{2}\left(\left|\tilde{\varepsilon}_{m}\right|+\mid \tilde{\varepsilon}_{m-l}\right)^{2}}, & \text { for } & (m-l+1 / 2)(m+1 / 2)>0 \\
\frac{-1}{\left|\tilde{\varepsilon}_{m-l}\right|^{2}\left(\left|\tilde{\varepsilon}_{m}\right|+\left|\tilde{\varepsilon}_{m-l}\right|\right)}, & \text { for } & (m-l+1 / 2)(m+1 / 2)<0
\end{array}\right\}\right]+O\left(\left(\frac{q}{k_{F}}\right)^{2}\right) \text {, }
\end{aligned}
$$

for $\omega_{l}>0$, where $N(0)$ is the density of states at the Fermi surface. Since $\bar{A}_{C=1}^{(c)}\left(\boldsymbol{q}, \omega_{q}=0 ; \omega_{l}\right)=O\left(\left(\frac{q}{k_{F}}\right)^{3}\right)$ and $\bar{S}_{C=1}^{(c)}\left(\boldsymbol{q}, \omega_{q}=\right.$ $\left.0 ; \omega_{l}\right)=O\left(\left(\frac{q}{k_{F}}\right)^{3}\right)$, the contribution from the (c) diagram is less singular near $T_{c}$ than that from (a) or (b). Therefore, we neglect the contributions from this diagram.

The most singular part in the dc limit for a clean system with large $\tau$ arises from the summation over $n$ in the region $n, m=0,1, \ldots, l-1$, where $\left|\tilde{\varepsilon}_{n}\right|+\left|\tilde{\varepsilon}_{n-l}\right|=\omega_{l}+1 / \tau$. We take only such terms and obtain

$$
\begin{aligned}
& \bar{A}_{C=1}^{(a)}\left(\boldsymbol{q}, \omega_{q}=0 ; \omega_{l}\right)=\frac{N(0)^{2} W_{0} q_{z}^{2}}{\left(\omega_{l}+1 / \tau\right)^{2}} \sum_{0 \leqslant n, m \leqslant l-1} \frac{1}{1+(2 \pi T / \Gamma)|n-m|} \\
& \times\left\{-\frac{5 \pi}{48} \frac{T v_{F}^{2}}{k_{F}^{2}} \frac{2 m-l+1}{(n-l+1 / 2-1 / 4 \pi \tau T)(m-l+1 / 2-1 / 4 \pi \tau T)}\right. \\
& \left.+\frac{1}{960 \pi} \frac{v_{F}^{4}}{T} \frac{2 m-l+1}{(n-l+1 / 2-1 / 4 \pi \tau T)^{2}(m-l+1 / 2-1 / 4 \pi \tau T)^{2}}\right\}, \\
& \bar{A}_{C=1}^{(b)}\left(\boldsymbol{q}, \omega_{q}=0 ; \omega_{l}\right)=\frac{N(0)^{2} W_{0} q_{z}^{2}}{\left(\omega_{l}+1 / \tau\right)^{2}} \sum_{0 \leqslant n, m \leqslant l-1} \frac{1}{1+(2 \pi T / \Gamma)|n-m|} \\
& \times\left\{-\frac{5 \pi}{48} \frac{T v_{F}^{2}}{k_{F}^{2}} \frac{2 m-l+1}{(n+1 / 2+1 / 4 \pi \tau T)(m-l+1 / 2-1 / 4 \pi \tau T)}\right. \\
& \left.+\frac{1}{960 \pi} \frac{v_{F}^{4}}{T} \frac{2 m-l+1}{(n+1 / 2+1 / 4 \pi \tau T)^{2}(m-l+1 / 2-1 / 4 \pi \tau T)^{2}}\right\}, \\
& \bar{S}_{C=1}^{(a)}\left(\boldsymbol{q}, \omega_{q}=0 ; \omega_{l}\right)=\frac{N(0)^{2} W_{0} q_{z}^{2}}{\left(\omega_{l}+1 / \tau\right)^{2}} \sum_{0 \leqslant n, m \leqslant l-1} \frac{1}{1+(2 \pi T / \Gamma)|n-m|} \frac{-1}{48 \pi} \\
& \times \frac{v_{F}^{3}}{k_{F} T} \frac{1}{(n-l+1 / 2-1 / 4 \pi \tau T)(m-l+1 / 2-1 / 4 \pi \tau T)^{2}}, \\
& \bar{S}_{C=1}^{(b)}\left(\boldsymbol{q}, \omega_{q}=0 ; \omega_{l}\right)=\frac{N(0)^{2} W_{0} q_{z}^{2}}{\left(\omega_{l}+1 / \tau\right)^{2}} \sum_{0 \leqslant n, m \leqslant l-1} \frac{1}{1+(2 \pi T / \Gamma)|n-m|} \frac{1}{48 \pi} \frac{v_{F}^{3}}{k_{F} T} \frac{1}{(n+1 / 2+1 / 4 \pi \tau T)(m-l+1 / 2-1 / 4 \pi \tau T)^{2}} .
\end{aligned}
$$


Up to now, we have calculated only the $C=1$ terms. By carrying out calculations similar to (B12)-(B33), we immediately find the $C=-1$ terms,

$$
\begin{aligned}
& \bar{A}_{C=-1}^{(i)}\left(\boldsymbol{q}, \omega_{q}=0 ; \omega_{l}\right)=-\bar{A}_{C=1}^{(i)}\left(\boldsymbol{q}, \omega_{q}=0 ; \omega_{l}\right), \\
& \bar{S}_{C=-1}^{(i)}\left(\boldsymbol{q}, \omega_{q}=0 ; \omega_{l}\right)=-\bar{S}_{C=1}^{(i)}\left(\boldsymbol{q}, \omega_{q}=0 ; \omega_{l}\right) .
\end{aligned}
$$

Now, we introduce $\bar{\alpha}_{C}^{(i)}(\boldsymbol{q}), \bar{\sigma}_{C}^{(i)}(\boldsymbol{q})(i=a, b$, and $c)$ defined as

$$
\bar{\alpha}_{C}^{(i)}(\boldsymbol{q})=\left.\frac{1}{T} \frac{\bar{A}_{C}^{(i)}\left(\boldsymbol{q}, \omega_{q}=0 ; \omega_{l}\right)}{\omega_{l}}\right|_{i \omega_{l} \rightarrow \omega+i 0}, \quad \bar{\sigma}_{C}^{(i)}(\boldsymbol{q})=\left.\frac{\bar{S}_{C}^{(i)}\left(\boldsymbol{q}, \omega_{q}=0 ; \omega_{l}\right)}{\omega_{l}}\right|_{i \omega_{l} \rightarrow \omega+i 0} .
$$

Then, we obtain

$$
\begin{gathered}
\bar{\alpha}_{C}^{(a)}(\boldsymbol{q})=C N(0)^{2} W_{0} \tau^{2} q_{z}^{2}\left[\frac{5\left\{\pi^{2} u_{(1,0)}(t, \gamma)-14 \zeta(3) \gamma u_{(1,1)}(t, \gamma)\right\}}{96} \frac{v_{F}^{2}}{k_{F}^{2} T}+\frac{\left\{-\pi^{4} u_{(2,1)}(t, \gamma)+\left[-\psi^{(4)}(1 / 2) / 6\right] \gamma u_{(2,2)}(t, \gamma)\right\}}{1920 \pi^{2}} \frac{v_{F}^{4}}{T^{3}}\right], \\
\bar{\alpha}_{C}^{(b)}(\boldsymbol{q})=C N(0)^{2} W_{0} \tau^{2} q_{z}^{2}\left[\frac{5\left\{-\pi^{2} u_{(1,0)}(t, \gamma)+\pi^{2} \gamma w_{(1,1)}(t, \gamma)\right\}}{96} \frac{v_{F}^{2}}{k_{F}^{2} T}+\frac{\left\{-2 \pi^{2} w_{(2,1)}(t, \gamma)+2 \pi^{2} \gamma w_{(2,2)}(t, \gamma)\right\}}{1920 \pi^{2}} \frac{v_{F}^{4}}{T^{3}}\right], \quad(\mathrm{B} 38) \\
\bar{\sigma}_{C}^{(a)}(\boldsymbol{q})=C \frac{\pi^{2} u_{(2,1)}(t, \gamma)}{192} \frac{v_{F}^{3}}{k_{F} T^{2}} N(0)^{2} W_{0} \tau^{2} q_{z}^{2}, \\
\bar{\sigma}_{C}^{(b)}(\boldsymbol{q})=C \frac{w_{(2,1)}(t, \gamma)}{96} \frac{v_{F}^{3}}{k_{F} T^{2}} N(0)^{2} W_{0} \tau^{2} q_{z}^{2},
\end{gathered}
$$

where $t=2 \pi T / \Gamma$ and $\gamma=1 / 2 \pi \tau T$, and the definitions of dimensionless functions $u_{(i, j)}(t, \gamma)$ and $w_{(i, j)}(t, \gamma)$ are given in Appendix D. In the clean limit $(\gamma \rightarrow 0)$,

$$
\begin{gathered}
\bar{\alpha}_{C}^{(a)}(\boldsymbol{q})=C N(0)^{2} W_{0} \tau^{2} q_{z}^{2}\left[\frac{5 \pi^{2} u_{(1,0)}\left(\frac{2 \pi T}{\Gamma}, 0\right)}{96} \frac{v_{F}^{2}}{k_{F}^{2} T}-\frac{\pi^{2} u_{(2,1)}\left(\frac{2 \pi T}{\Gamma}, 0\right)}{1920} \frac{v_{F}^{4}}{T^{3}}\right], \\
\bar{\alpha}_{C}^{(b)}(\boldsymbol{q})=C N(0)^{2} W_{0} \tau^{2} q_{z}^{2}\left[-\frac{5 \pi^{2} u_{(1,0)}\left(\frac{2 \pi T}{\Gamma}, 0\right)}{96} \frac{v_{F}^{2}}{k_{F}^{2} T}-\frac{w_{(2,1)}\left(\frac{2 \pi T}{\Gamma}, 0\right)}{960} \frac{v_{F}^{4}}{T^{3}}\right], \\
\bar{\sigma}_{C}^{(a)}(\boldsymbol{q})=C \frac{\pi^{2} u_{(2,1)}\left(\frac{2 \pi T}{\Gamma}, 0\right)}{192} \frac{v_{F}^{3}}{k_{F} T^{2}} N(0)^{2} W_{0} \tau^{2} q_{z}^{2}, \\
\bar{\sigma}_{C}^{(b)}(\boldsymbol{q})=C \frac{w_{(2,1)}\left(\frac{2 \pi T}{\Gamma}, 0\right)}{96} \frac{v_{F}^{3}}{k_{F} T^{2}} N(0)^{2} W_{0} \tau^{2} q_{z}^{2} .
\end{gathered}
$$

Finally, we complete the calculations of the Nernst and Hall conductivities, which are given by the integral over $\boldsymbol{q}$ :

$$
\begin{aligned}
\alpha_{x y}^{\text {Kubo }} & =\sum_{i=a, b, c}(-2 e T) \sum_{\boldsymbol{q} C= \pm 1} \tilde{L}_{C}^{\prime}\left(\boldsymbol{q}, \omega_{q}=0 ; H\right) \bar{\alpha}_{C}^{(i)}(\boldsymbol{q}), \\
\sigma_{x y} & =\sum_{i=a, b, c}\left(2 e^{2} T\right) \sum_{\boldsymbol{q} C= \pm 1} \tilde{L}_{C}^{\prime}\left(\boldsymbol{q}, \omega_{q}=0 ; H\right) \bar{\sigma}_{C}^{(i)}(\boldsymbol{q}),
\end{aligned}
$$

where the chirality-dependent part of the BPS is given by

$$
\tilde{L}_{C}^{\prime}\left(\boldsymbol{q}, \omega_{q} ; H\right)=C\left(5 e H / 4 k_{F}^{2} g\right)\left[L\left(\boldsymbol{q}, \omega_{q}\right)\right]^{2} .
$$

Using the expressions (B41)-(B44) and (C8), we encounter ultraviolet divergence in the calculation of (B45) and (B46). Then, we introduce the cutoff momentum $\Lambda$. It is appropriate to set $\Lambda$ as the same order as $1 / \xi$, where $\xi$ is the coherence length. The precise definition of $\xi$ in this paper is given by Eq. (C9) shown later. The reason for this choice of $\Lambda$ is that the expansion of $L^{-1}$ up to the second order of $\boldsymbol{q},(\mathrm{C} 8)$, is justified when $|\boldsymbol{q}|$ is sufficiently smaller than $1 / \xi$, and, then, $\tilde{L}_{C}^{\prime}$ rapidly decreases as $|\boldsymbol{q}|$ increases in the region $|\boldsymbol{q}|>1 / \xi$, because of higher-order terms, as discussed in Appendix C. Besides, in $|\boldsymbol{q}|<\Lambda$, the expressions (B41)-(B44) are also justified, since $\xi k_{F} \gg 1$ is satisfied in almost all superconductors (indeed, $\xi k_{F} \sim 50$ in $\mathrm{URu}_{2} \mathrm{Si}_{2}$ [46]). To simplify the expressions of the final results, we assume that the domain of the integral is anisotropic: $\sum_{i} a_{i} q_{i}^{2} \leqslant\left(a_{x}^{2} a_{z}^{3}\right)^{1 / 5} \Lambda^{2}$, where the 
numerical factors are given by $a_{x}=a_{y}=6 / 7$ and $a_{z}=9 / 7$. Then, we obtain

$$
\begin{aligned}
& \alpha_{x y \text { chiral }}^{\text {Kubo }}=\frac{f\left(\frac{2 \pi T}{\Gamma}\right)}{2304} \frac{\tau^{2} e^{2} W_{0} v_{F}^{4} \Lambda H}{\xi^{4} g k_{F}^{2} T^{2}}\left(1-\frac{3 \sqrt{\varepsilon} \arctan \left(\frac{\xi \Lambda}{\sqrt{\varepsilon}}\right)}{2 \xi \Lambda}+\frac{1}{2+2(\xi \Lambda)^{2} / \varepsilon}\right), \\
& \sigma_{x y \text { chiral }}=\frac{5 f\left(\frac{2 \pi T}{\Gamma}\right)}{1152} \frac{\tau^{2} e^{3} W_{0} v_{F}^{3} \Lambda}{\xi^{4} g k_{F}^{3} T}\left(1-\frac{3 \sqrt{\varepsilon} \arctan \left(\frac{\xi \Lambda}{\sqrt{\varepsilon}}\right)}{2 \xi \Lambda}+\frac{1}{2+2(\xi \Lambda)^{2} / \varepsilon}\right),
\end{aligned}
$$

in the clean limit, where $\xi^{\prime}=\left(a_{x}^{2} a_{z}^{3}\right)^{1 / 10} \approx 1.05 \xi$ and $f(t)=$ $u_{(2,1)}(t, 0)+\left(2 / \pi^{2}\right) w_{(2,1)}(t, 0)$. Since $\xi^{\prime} \sim \xi$, we use $\xi$ instead of $\xi^{\prime}$ in the following. For $T \sim T_{c}$, the above equations are reduced to Eqs. (4) and (5) in the main text.

\section{APPENDIX C: MOMENTUM REPRESENTATION OF FLUCTUATION PROPAGATORS UNDER ZERO MAGNETIC FIELD}

In this section, we derive the expression of the fluctuation propagator of $d_{z x} \pm i d_{z y}$-wave superconductors under zero magnetic field, which is used in Appendix B. It is given by

$$
\begin{gathered}
L^{-1}\left(\boldsymbol{q}, \omega_{q}\right)=-1 / g+\Pi\left(\boldsymbol{q}, \omega_{q}\right), \\
\Pi\left(\boldsymbol{q}, \omega_{q}\right)=T \sum_{n} \Pi_{\varepsilon_{n}}\left(\boldsymbol{q}, \omega_{q}\right), \\
\Pi_{\varepsilon_{n}}\left(\boldsymbol{q}, \omega_{q}\right)=\sum_{\boldsymbol{k}}|\phi(\boldsymbol{k})|^{2} G\left(\boldsymbol{k}+\frac{\boldsymbol{q}}{2}, \varepsilon_{n+q}\right) \\
\times G\left(-\boldsymbol{k}+\frac{\boldsymbol{q}}{2},-\varepsilon_{n}\right),
\end{gathered}
$$

where $\quad G\left(\boldsymbol{k}, \varepsilon_{k}\right)^{-1}=i \tilde{\varepsilon}_{k}-\xi_{\boldsymbol{k}}=i\left[\varepsilon_{k}+\operatorname{sgn}\left(\varepsilon_{\mathrm{k}}\right) / 2 \tau\right]-\xi_{\boldsymbol{k}}$, $\phi(\boldsymbol{k})=\sqrt{15 / 2} k_{z}\left(k_{x}+i k_{y}\right) / k_{F}^{2}$. Now, replacing the sum with the integral over the energy and average over the Fermi surface, $\langle\cdots\rangle_{\hat{k}}$, we obtain

$\Pi_{\varepsilon_{n}}\left(\boldsymbol{q}, \omega_{q}\right)=2 \pi N(0) \theta\left(\varepsilon_{n+q} \varepsilon_{n}\right)\left\langle\frac{|\phi(\boldsymbol{k})|^{2}}{\left|\tilde{\varepsilon}_{n+q}+\tilde{\varepsilon}_{n}\right|+i \Delta \xi(\boldsymbol{k}, \boldsymbol{q})}\right\rangle_{\hat{\boldsymbol{k}}}$,

where $N(0)$ is the density of state at the Fermi surface, $\theta$ is the Heaviside step function, and $\Delta \xi(\boldsymbol{k}, \boldsymbol{q})=\xi_{\boldsymbol{k}+\boldsymbol{q} / 2}-\xi_{\boldsymbol{k}-\boldsymbol{q} / 2}$. Expanding it with respect to $\boldsymbol{q}$, we obtain the expression up to the quadratic term:

$$
\begin{aligned}
& \Pi_{\varepsilon_{n}}\left(\boldsymbol{q}, \omega_{q}\right) \\
& =\frac{2 \pi N(0) \theta\left(\varepsilon_{n+q} \varepsilon_{n}\right)}{\left|\tilde{\varepsilon}_{n+q}+\tilde{\varepsilon}_{n}\right|}\left[\left\langle|\phi(\boldsymbol{k})|^{2}\right\rangle_{\hat{\boldsymbol{k}}}-\frac{\left\langle|\phi(\boldsymbol{k})|^{2}\left(\boldsymbol{v}_{\boldsymbol{k}} \cdot \boldsymbol{q}\right)^{2}\right\rangle_{\hat{\boldsymbol{k}}}}{\left|\tilde{\varepsilon}_{n+q}+\tilde{\varepsilon}_{n}\right|^{2}}\right] \\
& =\frac{2 \pi N(0) \theta\left(\varepsilon_{n+q} \varepsilon_{n}\right)}{\left|\tilde{\varepsilon}_{n+q}+\tilde{\varepsilon}_{n}\right|}\left[1-\frac{v_{F}^{2} \sum_{i=x, y, z} a_{i} q_{i}^{2}}{\left|\tilde{\varepsilon}_{n+q}+\tilde{\varepsilon}_{n}\right|^{2}}\right],
\end{aligned}
$$

where $v_{F}$ is the Fermi velocity and $a_{x}=a_{y}=6 / 7$ and $a_{z}=9 / 7$ are numerical factors which reflect anisotropy of $V^{ \pm}\left(\boldsymbol{k}, \boldsymbol{k}^{\prime}\right)$. Here, this expansion is justified when $\Delta \xi(\boldsymbol{k}, \boldsymbol{q})$ is sufficiently smaller than $\left|\tilde{\varepsilon}_{n+q}+\tilde{\varepsilon}_{n}\right|$. Due to the factor $\theta\left(\varepsilon_{n+q} \varepsilon_{n}\right),\left|\tilde{\varepsilon}_{n+q}+\tilde{\varepsilon}_{n}\right|$ is equal to or larger than $2 \pi T$, and, therefore, this condition is read as $v_{F} q \ll T \Longleftrightarrow q \ll 1 / \xi$, where the coherence length $\xi$ used in this paper is precisely defined by Eq. (C9).

Now, we take the sum over $n$, in which we introduce the cutoff energy $\omega_{D}$ for the pairing interaction and the upper limit of the frequency sum $N_{\max }=\omega_{D} / 2 \pi T$ to remove the ultraviolet logarithmic divergence of the first sum:

$$
\begin{aligned}
\frac{\Pi\left(\boldsymbol{q}, \omega_{q}\right)}{N(0)}= & \psi\left(\frac{1}{2}+\frac{\left|\omega_{q}\right|}{4 \pi T}+\frac{\omega_{D}}{2 \pi T}+\frac{1}{4 \pi T \tau}\right) \\
& -\psi\left(\frac{1}{2}+\frac{\left|\omega_{q}\right|}{4 \pi T}+\frac{1}{4 \pi T \tau}\right) \\
& +\frac{v_{F}^{2} a_{i} q_{i}^{2}}{2(4 \pi T)^{2}} \psi^{\prime \prime}\left(\frac{1}{2}+\frac{1}{4 \pi T \tau}\right) .
\end{aligned}
$$

The superconducting transition temperature $T_{c}$ is defined by $\left.L^{-1}(\mathbf{0}, 0)\right|_{T=T_{c}}=0$, which is rewritten into

$$
\psi\left(\frac{1}{2}+\frac{\omega_{D}}{2 \pi T}+\frac{1}{4 \pi T_{c} \tau}\right)-\psi\left(\frac{1}{2}+\frac{1}{4 \pi T_{c} \tau}\right)=\frac{1}{g N(0)} .
$$

Therefore, we obtain the expression for the fluctuation propagator in the vicinity of $T_{c}$, i.e., $\varepsilon=\ln T / T_{c} \ll 1$ :

$$
\begin{aligned}
L^{-1}\left(\boldsymbol{q}, \omega_{q}\right)= & -N(0)\left[\varepsilon+\psi\left(\frac{1}{2}+\frac{\left|\omega_{q}\right|}{4 \pi T}+\frac{1}{4 \pi T \tau}\right)\right. \\
& \left.-\psi\left(\frac{1}{2}+\frac{1}{4 \pi T \tau}\right)+\sum_{i} \xi_{i}^{2}(\tau) q_{i}^{2}\right],
\end{aligned}
$$

where the coherence length is given by $\xi_{i}^{2}(\tau)=a_{i} \xi^{2}(\tau)$, where $\xi^{2}(\tau)=-v_{F}^{2} \psi^{\prime \prime}(1 / 2+1 / 4 \pi T \tau) / 6(4 \pi T)^{2}$. The coherence length used in this paper is defined by

$$
\xi=\xi(\tau=\infty) \text {. }
$$

Equation (C8) is the main result of this section.

\section{APPENDIX D: DIMENSIONLESS FUNCTIONS}

In this section, we give the definitions of the dimensionless functions which appear in the formulas of the Nernst and Hall conductivities presented in the previous section. We also obtain their approximated but explicit expressions. The main result of this section is that the dimensionless function $f(t)$ which appears in Eqs. (4) and (5) in the main text is well approximated by a smooth function $\bar{f}^{\text {app }}(t)$ (D17) for temperature regions where superconducting fluctuations are strong. We use it for the numerical calculation of temperature dependencies of transport coefficients shown in Fig. 3 in the main text. 


\section{Definitions}

The definitions of dimensionless functions which appear in Eqs. (B37)-(B40) are

$$
\begin{aligned}
& u_{(i, j)}(t, \gamma)=\frac{(-1)^{i+j+1}(i+j-1) !}{\psi^{(i+j)}\left(\frac{1}{2}\right)}\left[\frac{2 \pi T}{\omega_{l}} \sum_{0 \leqslant n, m \leqslant l-1} \frac{1}{\left(n+\frac{1}{2}+\frac{\gamma}{2}\right)^{i}\left(m+\frac{1}{2}+\frac{\gamma}{2}\right)^{j}(1+t|n-m|)}\right] \begin{array}{c}
i \omega_{l} \rightarrow \omega+i 0, \\
\omega \rightarrow 0
\end{array}, \\
& w_{(i, j)}(t, \gamma)=\frac{(i-1) !(j-1) !}{(i+j-2) ! \pi^{2}}\left[\frac{2 \pi T}{\omega_{l}} \sum_{0 \leqslant n, m \leqslant l-1} \frac{1}{\left(n+\frac{1}{2}+\frac{\gamma}{2}\right)^{i}\left(l-m-\frac{1}{2}+\frac{\gamma}{2}\right)^{j}(1+t|n-m|)}\right] \begin{array}{c}
i \omega_{l} \rightarrow \omega+i 0, \\
\omega \rightarrow 0
\end{array},
\end{aligned}
$$

where $(i, j)$ are nonnegative integers and the domains of definitions are $i+j \geqslant 1$ for $u_{(i, j)}(t)$, and $i, j \geqslant 1$ for $w_{(i, j)}(t, \gamma)$. An important property of these functions is that at $t=0$,

$$
u_{(i, j)}(t=0, \gamma)=w_{(i, j)}(t=0, \gamma)=0 .
$$

Also, their normalization factors are determined by the following conditions:

$$
\begin{gathered}
u_{(i, j)}(t=\infty, \gamma=0)=1, \\
w_{(i, j)}(t=\infty, \gamma)=\frac{1}{\gamma^{i+j-1}}+O\left(\frac{1}{\gamma^{i+j-2}}\right) \quad \text { as } \gamma \rightarrow 0 .
\end{gathered}
$$

Equations (D3)-(D5) are proved in Appendix D 3.

The dimensionless function $f(t)$ which appears in Eqs. (4) and (5) in the main text and in Eqs. (B48) and (B49) is defined by using $u_{(i, j)}(t, \gamma)$ and $w_{(i, j)}(t, \gamma)$ as

$$
f(t)=u_{(2,1)}(t, 0)+\left(2 / \pi^{2}\right) w_{(2,1)}(t, 0) .
$$

\section{Approximation functions}

We here introduce analytically solvable approximation functions for the dimensionless functions (D1) and (D2). They are defined by the analytic continuation of functions of the Matsubara frequencies:

$$
\begin{gathered}
u^{\mathrm{app}}(t)=\left.\frac{2 \pi T}{\omega_{l}} \sum_{0 \leqslant n, m \leqslant l-1} \frac{1}{1+t|n-m|}\right|_{\substack{i \omega_{l} \rightarrow \omega+i 0, \omega \rightarrow 0}}, \\
w_{i+j}^{\mathrm{app}}(t, \gamma)=\left.\frac{2 \pi T}{\omega_{l}} \sum_{0 \leqslant n, m \leqslant l-1} \frac{1}{(n-m+l+\gamma)^{i+j-1}} \frac{1}{1+t|n-m|}\right|_{\substack{i \omega_{l} \rightarrow \omega+i 0, \omega \rightarrow 0}},
\end{gathered}
$$

and they can be rewritten into compact expressions:

$$
\begin{aligned}
& u^{\mathrm{app}}(t)=-1-\frac{2}{t}+\frac{2}{t^{2}} \psi^{\prime}\left(\frac{1}{t}\right), \\
& w_{i+j}^{\mathrm{app}}(t, \gamma)=\frac{(-1)^{i+j-2}}{(i+j-2) !} \frac{\partial^{i+j-2}}{\partial \gamma^{i+j-2}} w_{2}^{\mathrm{app}}(t, \gamma), \\
& w_{2}^{\mathrm{app}}(t, \gamma)= \frac{1}{(1 / t-\gamma) t}\left[\gamma \psi^{\prime}(\gamma)-\frac{1}{t} \psi^{\prime}(1 / t)\right] \\
&-\frac{1}{(1 / t+\gamma) t}\left[\gamma \psi^{\prime}(1+\gamma)-\frac{1}{t} \psi^{\prime}(1 / t)\right]-\frac{1}{\gamma},
\end{aligned}
$$

which are derived in Appendix D 3.

We can expect that $u^{\text {app }}(t)$ and $w_{i+j}^{\text {app }}(t, \gamma)$ with small $\gamma$ are good approximation functions for $u_{(i, j)}(t, \gamma=0)$ and $w_{(i, j)}(t, \gamma)$, respectively, because of the following reason. They have the same asymptotic behaviors as the original functions for $t \rightarrow \infty$, and also take the same values as the original ones at $t=0$ :

$$
\left.\frac{\partial^{n} u_{(i, j)}(t, \gamma=0)}{\partial t^{n}}\right|_{t=\infty}=\left.\frac{\partial^{n} u^{\mathrm{app}}(t)}{\partial t^{n}}\right|_{t=\infty}=\delta_{n, 0} \quad \text { for } n \geqslant 0,
$$

$$
u_{(i, j)}(t=0, \gamma)=u^{\mathrm{app}}(t=0)=0
$$

and

$$
\begin{aligned}
w_{(i, j)}(t=\infty, \gamma) & =w_{i+j}^{\mathrm{app}}(t=\infty, \gamma) \\
& =\frac{1}{\gamma^{i+j-1}}+O\left(\frac{1}{\gamma^{i+j-2}}\right) \quad \text { as } \gamma \rightarrow 0
\end{aligned}
$$




$$
\begin{aligned}
\left.\frac{\partial^{n} w_{(i, j)}(t, \gamma)}{\partial t^{n}}\right|_{\gamma: \text { fixed }, t=\infty} & =\left.\frac{\partial^{n} w_{i+j}^{\text {app }}(t, \gamma)}{\partial t^{n}}\right|_{\gamma: \text { fixed }, t=\infty} \\
& =0, \quad \text { for } n \geqslant 1,
\end{aligned}
$$

$$
w_{(i, j)}(t=0, \gamma)=w_{i+j}^{\mathrm{app}}(t=0, \gamma)=0 \text {. }
$$

We will prove these relations in Appendix D 3 .

In Fig. 6, we plot these approximation functions (dashed line) and numerical estimations of Eqs. (D1) and (D2) (dots) which are obtained by the Padé method. In this plot, we focus on $u_{(2,1)}(t, 0), w_{(2,1)}(t, 0)$, and their sum, $f(t)=u_{(2,1)}(t, 0)+$ $\left(2 / \pi^{2}\right) w_{(2,1)}(t, 0)$, which appears in Eqs. (4) and (5). Here, the range of the plot is set to be $0<t<2.0$, which covers the region of superconducting fluctuations $t_{c}<t<x t_{c}, x \sim 3$, with $t_{c}=2 \pi T_{c} / \Gamma=0.5$. In these calculations, we used the material parameters of $\mathrm{URu}_{2} \mathrm{Si}_{2}$, i.e., $T_{c} \sim 1.5 \mathrm{~K}$ and $\Gamma \sim$ $1.5 \mathrm{meV}[16,35]$. As seen from Fig. 6, the $t$ dependencies of the approximation functions are qualitatively similar to the original functions. However, there are slight quantitative differences. Then, to improve the approximation functions, we scale them as

$$
\begin{gathered}
\bar{u}^{\mathrm{app}}(t)=c_{u} u^{\mathrm{app}}\left(c_{u}^{\prime} t\right), \\
\bar{w}_{3}^{\mathrm{app}}(t, 0)=c_{w} w_{3}^{\mathrm{app}}\left(c_{w}^{\prime} t, 0\right), \\
\bar{f}^{\mathrm{app}}(t)=\bar{u}^{\mathrm{app}}(t)+\frac{2}{\pi^{2}} \bar{w}_{3}^{\mathrm{app}}(t, 0),
\end{gathered}
$$

where the scaling parameter constants are obtained by fitting the numerical data, and we find $c_{u}=0.72, c_{u}^{\prime}=0.91, c_{w}=$ 0.76 , and $c_{w}^{\prime}=1.14$. The improved approximation functions are also shown in Fig. 6 (solid line) and we see that they coincide with the original functions quite well. Therefore, we use these smooth functions to calculate temperature dependencies of transport coefficients in the main text.
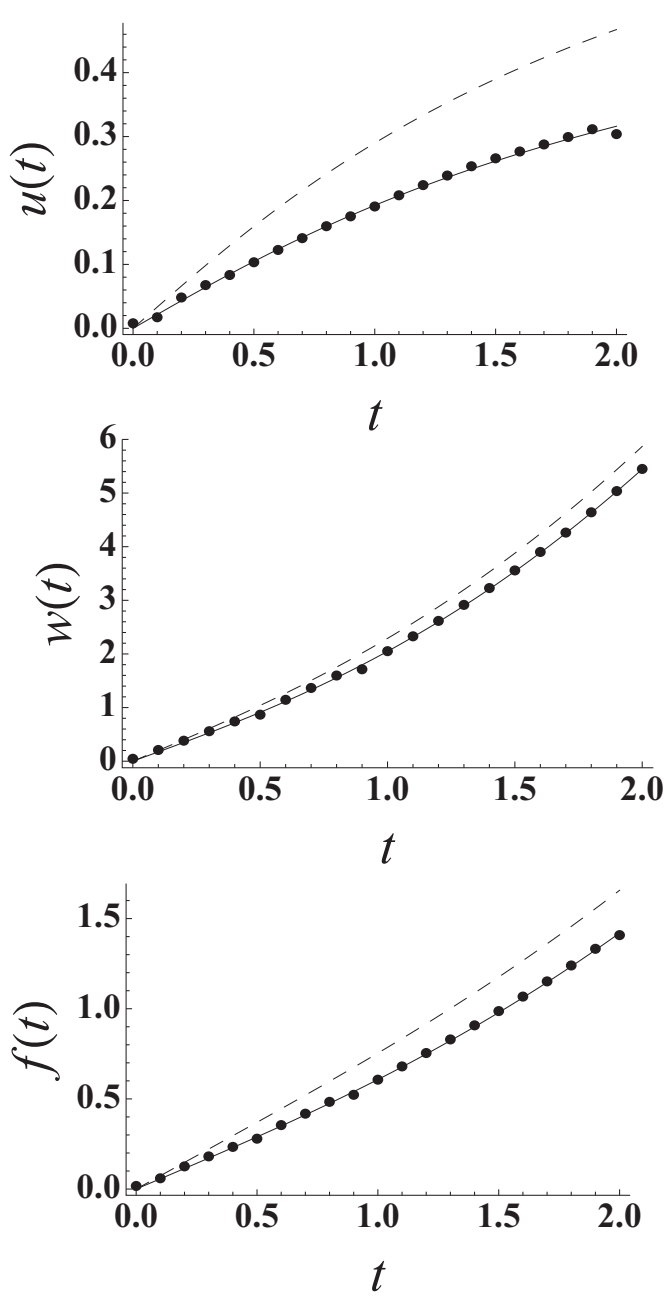

FIG. 6. Numerical result for $u_{(2,1)}(t, 0), w_{(2,1)}(t, 0), f(t)$ (dots), approximation function $u^{\mathrm{app}}(t), w_{3}^{\mathrm{app}}(t, 0), f^{\mathrm{app}}(t)$ (dashed line), and modified approximation function $\bar{u}^{\text {app }}(t), \bar{w}_{3}^{\text {app }}(t, 0), \bar{f}^{\text {app }}(t)$ (solid line). The first and third ones coincide with each other quite well.

\section{Proofs of relations}

In this subsection, we give proofs of relations used in the previous sections.

\section{a. Proofs of Eq. (D3)}

We can easily verify the following relation,

$$
\begin{aligned}
& u_{(i, j)}(t=0, \gamma)=\left.c_{i j} \frac{2 \pi T}{\omega_{l}} \sum_{0 \leqslant n, m \leqslant l-1} \frac{1}{\left(n+\frac{1}{2}+\frac{\gamma}{2}\right)^{i}\left(m+\frac{1}{2}+\frac{\gamma}{2}\right)^{j}}\right|_{\substack{i \omega_{l} \rightarrow \omega+i 0, \omega \rightarrow 0}} \\
& =c_{i j}\left[\frac{2 \pi T}{\omega_{l}} \frac{\psi^{(i-1)}\left(\frac{1}{2}\right)-\psi^{(i-1)}\left(\frac{1}{2}+\frac{\omega_{l}}{2 \pi T}\right)}{(-1)^{i}(i-1) !} \frac{\psi^{(i-1)}\left(\frac{1}{2}\right)-\psi^{(i-1)}\left(\frac{1}{2}+\frac{\omega_{l}}{2 \pi T}\right)}{(-1)^{i}(i-1) !}\right] \begin{array}{c}
i \omega_{l} \rightarrow \omega+i 0, \\
\omega \rightarrow 0
\end{array} \\
& =0 \text {, }
\end{aligned}
$$

where $c_{i j}=(-1)^{i+j+1}(i+j-1) ! / \psi^{(i+j)}\left(\frac{1}{2}\right)$. Similar calculation leads to $w_{(i, j)}(t, \gamma)=0$. Then Eqs. (D3) is proved.

\section{b. Proofs of Eqs. (D4) and (D5)}

First, we prove Eq. (D4). Owing to the factor $1 /(1+t \mid n-$ $m \mid$ ), only terms satisfying $n=m$ contribute to the sum at 
$t \rightarrow \infty$. Therefore,

$$
\begin{aligned}
u_{(i, j)}(t=\infty, \gamma=0) & =\left.c_{i j} \frac{2 \pi T}{\omega_{l}} \sum_{n=0,1, \cdots, l-1} \frac{1}{\left(n+\frac{1}{2}\right)^{i+j}}\right|_{\substack{i \omega l \rightarrow \omega+i 0, \omega \rightarrow 0}} \\
& =\left.c_{i j} \frac{2 \pi T}{\omega_{l}} \frac{\psi^{(i+j-1)}\left(\frac{1}{2}\right)-\psi^{(i+j-1)}\left(\frac{1}{2}+\frac{\omega_{l}}{2 \pi T}\right)}{(-1)^{i+j}(i+j-1) !}\right|_{\substack{i \omega_{l} \rightarrow \omega+i 0, \omega \rightarrow 0}}=c_{i j} / c_{i j}=1 .
\end{aligned}
$$

Next, we prove Eq. (D5). Taking the limit $t \rightarrow \infty$, we have

$$
w_{(i, j)}(t=\infty, \gamma)=\left.\frac{(i-1) !(j-1) !}{(i+j-2) ! \pi^{2}} \frac{2 \pi T}{\omega_{l}} \sum_{n=0,1, \cdots, l-1} \frac{1}{\left(n+\frac{1}{2}+\frac{\gamma}{2}\right)^{i}\left(l-n-\frac{1}{2}+\frac{\gamma}{2}\right)^{j}}\right|_{\substack{i \omega_{l} \rightarrow \omega+i 0, \omega \rightarrow 0}} .
$$

Now, let

$$
\tilde{w}_{(i, j)}(\gamma)=\left.\frac{2 \pi T}{\omega_{l}} \sum_{n=0,1, \cdots, l-1} \frac{1}{\left(n+\frac{1}{2}+\frac{\gamma}{2}\right)^{i}\left(l-n-\frac{1}{2}+\frac{\gamma}{2}\right)^{j}}\right|_{\substack{i \omega_{l} \rightarrow \omega+i 0 \\ \omega \rightarrow 0}} .
$$

Then,

$$
\begin{aligned}
\tilde{w}_{(i, j)}(\gamma) \frac{1}{\gamma}\left[\tilde{w}_{(i, j-1)}(\gamma)+\tilde{w}_{(i-1, j)}(\gamma)\right]= & \frac{1}{\gamma^{2}}\left[\tilde{w}_{(i, j-2)}(\gamma)+2 \tilde{w}_{(i-1, j-1)}(\gamma)+\tilde{w}_{(i-2, j)}(\gamma)\right] \\
& \vdots \\
= & \frac{(i+j-2) !}{(i-1) !(j-1) !} \frac{1}{\gamma^{i+j-1}}\left[\tilde{w}_{(1,0)}(\gamma)+\tilde{w}_{(0,1)}(\gamma)\right]+c_{1}^{\prime} \frac{1}{\gamma^{i+j-2}}\left[\tilde{w}_{(2,0)}(\gamma)+\tilde{w}_{(0,2)}(\gamma)\right] \\
& +c_{2}^{\prime} \frac{1}{\gamma^{i+j-3}}\left[\tilde{w}_{(3,0)}(\gamma)+\tilde{w}_{(0,3)}(\gamma)\right]+\cdots
\end{aligned}
$$

where $c_{1}^{\prime}, c_{2}^{\prime}, \ldots$ are constants. By using

$$
\tilde{w}_{(i, 0)}(\gamma=0)=\left.\frac{2 \pi T}{\omega_{l}} \sum_{n=0,1, \cdots, l-1} \frac{1}{\left(n+\frac{1}{2}\right)^{i}}\right|_{\substack{i \omega_{l} \rightarrow \omega+i 0, \omega \rightarrow 0}}=\frac{(-1)^{i+1} \psi^{(i)}\left(\frac{1}{2}\right)}{(i-1) !},
$$

we obtain

$$
w_{(i, j)}(t=\infty, \gamma)=\frac{(i-1) !(j-1) !}{(i+j-2) ! \pi^{2}} \tilde{w}_{(i, j)}(\gamma)=\frac{1}{\gamma^{i+j-1}}+O\left(\frac{1}{\gamma^{i+j-2}}\right) \quad \text { as } \quad \gamma \rightarrow 0 .
$$

Then, the normalization conditions (D4) and (D5) are proved.

\section{c. Proofs of Eqs. (D8) and (D9)}

Now, introducing $N=n-m$ and dividing the region of summation in (D6) into $\sum_{n \leqslant m}+\sum_{n \geqslant m}-\sum_{n=m}$, we obtain

$$
\begin{aligned}
& u^{\mathrm{app}}(t)=\left.2\left[\frac{2 \pi T}{\omega_{l}} \sum_{N=0,1, \ldots, l-1} \frac{l-N}{1+t N}\right]\right|_{\substack{i \omega_{l} \rightarrow \omega+i 0, \omega \rightarrow 0}}-1 \\
& =-1-\frac{2}{t}+\left.\frac{2}{t^{2}}\left[\frac{2 \pi T}{\omega_{l}} \sum_{N=0,1, \ldots, l-1} \frac{1}{N+1 / t}\right]\right|_{\substack{i \omega_{l} \rightarrow \omega+i 0, \omega \rightarrow 0}}+\left.\frac{2}{t}\left[\sum_{N=0,1, \ldots, l-1} \frac{1}{1+t N}\right]\right|_{\substack{i \omega_{l} \rightarrow \omega+i 0, \omega \rightarrow 0}} \\
& =-1-\frac{2}{t}+\frac{2}{t^{2}} \psi^{\prime}\left(\frac{1}{t}\right) .
\end{aligned}
$$

From it we can derive Eq. (D8). Furthermore, Eq. (D9) can be obtained by similar calculations. 


\section{d. Proofs of Eqs. (D10) and (D11)}

First, we prove Eq. (D10). The $n=0$ case immediately follows from Eq. (D4) and the explicit expression for $u_{\text {app }}(t)$, (D8). For $n \geqslant 1$,

$$
\begin{aligned}
& \left.\frac{\partial^{n} u_{(i, j)}(t, \gamma=0)}{\partial t^{n}}\right|_{t=\infty} \\
& =\left.c_{i j} \frac{2 \pi T}{\omega_{l}} \sum_{0 \leqslant a, b \leqslant l-1} d_{i j}^{a b}(-1)^{n} n ! \frac{|a-b|^{n}}{(1+t|a-b|)^{n+1}}\right|_{\substack{i \omega_{l} \rightarrow \omega+i 0, \omega \rightarrow 0}},
\end{aligned}
$$

where $d_{i j}^{a b}=1 /\left(a+\frac{1}{2}\right)^{i}\left(b+\frac{1}{2}\right)^{j}$. Now, owing to the factor $1 /(1+t|a-b|)^{n+1}$, the contributions from the terms satisfying $a \neq b$ become zero in $t \rightarrow \infty$ limit. Moreover, the other terms, which satisfy $a=b$, are also zero for $n \geqslant 1$ on account of the factor $|a-b|^{n}$. Therefore, we find that Eq. (D26) is zero in the $t \rightarrow \infty$ limit. A similar calculation leads to $\partial^{n} u^{\text {app }}(t) /\left.\partial t^{n}\right|_{t=\infty}=0$, which also directly follows from the explicit expression (D8). Then, Eq. (D10) for any $n \geqslant 0$ is proven.

On the other hand, Eq. (D11) instantly follows from Eq. (D3) and a calculation similar to Eq. (D18).

\section{e. Proofs of Eqs. (D12), (D13), and (D14)}

The equivalence between the left-hand side and right-hand side of Eq. (D12) has been already proven (D5). Then, we now prove the equivalence between the middle one and the right-hand side. Owing to the factor $1 /(1+t|n-m|)$, only terms satisfying $n=m$ contribute to the sum at $t \rightarrow \infty$ :

$$
\begin{aligned}
& w_{i+j}^{\text {app }}(t=\infty, \gamma) \\
& =\left.\frac{2 \pi T}{\omega_{l}} \sum_{n=0,1, \cdots, l-1} \frac{1}{(l+\gamma)^{i+j-1}}\right|_{\substack{i \omega_{l} \rightarrow \omega+i 0, \omega \rightarrow 0}} \\
& =\frac{1}{\gamma^{i+j-1}} .
\end{aligned}
$$

Then, the whole of Eq. (D12) is proven.

On the other hand, Eqs. (D13) and (D14) can be derived with techniques similar to those used in the derivations of $\mathrm{Eq}$ (D10) for $n \geqslant 1$ and Eq. (D11).

\section{APPENDIX E: MAGNETIZATION}

In this section we discuss the magnetization and derive Eq. (6) in the main text. In this paper $\hat{A}$ means a matrix whose element is $A(\boldsymbol{x}, \boldsymbol{y})$ and $\hat{1}_{\boldsymbol{x}, \boldsymbol{y}}=\delta(\boldsymbol{x}-\boldsymbol{y})$. The free energy in the presence of the magnetic field, $\boldsymbol{H}=(0,0, H)$, is given by

$$
\begin{aligned}
F[H] & =T \sum_{\omega_{q}, C= \pm 1} \operatorname{Tr} \ln \left[-\hat{\tilde{L}}_{C}^{-1}\left(\omega_{q} ; H\right)\right] \\
& =2 T \sum_{\omega_{q}} \operatorname{Tr} \ln \left[\hat{1}-g e^{-2 i \hat{\Phi}} \hat{\Pi}\left(\omega_{q} ; H\right)\right]+T \sum_{\omega_{q}} \operatorname{Tr} \ln \left[\hat{1}-\left(\frac{5 e H g}{4 k_{F}^{2}}\right)^{2} \frac{e^{-2 i \hat{\Phi}} \hat{\Pi}^{\prime 2}\left(\omega_{q} ; H\right)}{\left[1-g e^{-2 i \hat{\Phi}} \hat{\Pi}\left(\omega_{q} ; H\right)\right]^{2}}\right] .
\end{aligned}
$$

Then, the magnetic susceptibility is given as

$$
\chi=\chi_{\text {dia }}+\chi_{\text {chiral }}
$$

where

$$
\chi_{\text {dia }}=-\left.\frac{\partial^{2}}{\partial H^{2}}\left\{2 T \sum_{\omega_{q}} \operatorname{Tr} \ln \left[\hat{1}-g e^{-2 i \hat{\Phi}} \hat{\Pi}\left(\omega_{q} ; H\right)\right]\right\}\right|_{H \rightarrow 0}
$$

is the fluctuation diamagnetism term which appears also in nonchiral superconductors [37], where the factor 2 reflects the fact that the number of fluctuation channels is two, and

$$
\begin{aligned}
\chi_{\text {chiral }} & =-\left.\frac{\partial^{2}}{\partial H^{2}}\left\{T \sum_{\omega_{q}} \operatorname{Tr} \ln \left[\hat{1}-\frac{\left(\frac{5 e H g}{4 k_{F}^{2}}\right)^{2} e^{-2 i \hat{\Phi}} \hat{\Pi}^{\prime 2}\left(\omega_{q} ; H\right)}{\left(\hat{1}-g e^{-2 i \hat{\Phi}} \hat{\Pi}\left(\omega_{q} ; H\right)\right)^{2}}\right]\right\}\right|_{H \rightarrow 0} \\
& =2 T\left(\frac{5 e g}{4 k_{F}^{2}}\right)^{2} \sum_{\omega_{q}} \operatorname{Tr}\left[\frac{\hat{\Pi}^{\prime 2}\left(\omega_{q} ; H=0\right)}{\left(\hat{1}-g \hat{\Pi}\left(\omega_{q} ; H=0\right)\right)^{2}}\right]
\end{aligned}
$$

is the contribution unique to chiral superconductors. Now, we evaluate the chirality-induced term $(\mathrm{E} 4)$. Neglecting the $\omega_{q} \neq 0$ terms, which are less singular in the vicinity of $T_{c}$ than the $\omega_{q}=0$ one, we obtain

$$
\chi_{\text {chiral }}=\frac{25 e^{2} T}{64 \pi k_{F}^{4} \xi^{3}(N(0) g)^{2} \varepsilon^{1 / 2}}>0,
$$

which is positive, indicating the paramagnetic response due to $\mathrm{MC}$ coupling. In this calculation, we used the momentum representation of the BPS, (C8). 


\section{APPENDIX F: CALCULATION SCHEME OF FIGURE 3}

In this section, we explain the calculation scheme of the temperature dependence of the CSF-induced Nernst conductivity $\alpha_{x y}$ shown in Fig. 3 in the main text. For the function $f(t)$, we used the approximation function $\bar{f}^{\text {app }}(t)$ (D17). We also used the material parameters of $\mathrm{URu}_{2} \mathrm{Si}_{2}: T_{c} \sim$ $1.5 \mathrm{~K}, k_{F} \sim 0.5 \times 10^{10} \mathrm{~m}^{-1}, v_{F} \sim 1 \times 10^{4} \mathrm{~m} / \mathrm{s}$ [46], and $\Gamma \sim 1.5 \mathrm{meV}[35,36]$, and set the cutoff momentum as $\Lambda=1 / \xi$. We neglected the $\tau$ dependence of the transition temperature $T_{c}$ and coherence length $\xi$. These assumptions are justified for $T \tau \gg 1$. In this paper, RRR is defined as the ratio of $\sigma_{x x}(T=300 \mathrm{~K})$ to $\sigma_{x x}(T=0 \mathrm{~K})$, which is obtain by extrapolating the temperature dependence of the conductivity in the normal-metal region to $T=0 \mathrm{~K}$. It is also assumed that the longitudinal conductivity obeys $\sigma_{x x} \propto \tau^{-1}=\tau_{\text {imp }}^{-1}+A T^{2}$, where $\tau_{\text {imp }}$ and $A$ are $T$-independent constants.

\section{APPENDIX G: HOW THE RESULTS DEPEND ON THE SPECIFIC FORM OF $W\left(k, \omega_{j}\right)$, SPATIAL DIMENSIONALITY, AND THE PAIRING SYMMETRY}

In this section, we discuss to what extent the main results, (4) and (5), depend on the functional form of the renormalized four-point vertex, $W\left(\boldsymbol{k}, \omega_{j}\right)$, the spatial dimensionality, and the pairing symmetry of chiral superconducting states.

Here, we summarize the main results of this section: (1) The result that $\alpha_{x y \text { chiral }}^{\text {Kubo }}$ and $\sigma_{x y \text { chiral }}$ are proportional to $\tau^{2}$ is not changed by these three conditions. (2) The critical behavior may be changed by the dimensionality and the pairing symmetry, but not by the functional form of $W\left(\boldsymbol{k}, \omega_{j}\right)$. (3) The magnitudes of $\alpha_{x y \text { chiral }}^{\text {Kubo }}$ and $\sigma_{x y \text { chiral }}$ depend on the specific form of $W\left(\boldsymbol{k}, \omega_{j}\right)$ : they are decreased as the range of momentum transfer raised by the interaction $W\left(\boldsymbol{k}, \omega_{j}\right)$ becomes narrower. In the succeeding subsections, we will discuss the details.

\section{1. $\tau$ dependence}

Irrespective of functional forms of $W\left(\boldsymbol{k}, \omega_{j}\right)$, spatial dimensionality, and the pairing symmetry of chiral superconducting states, $\bar{A}_{C}^{(i)}\left(\boldsymbol{q}, \omega_{q} ; \omega_{l}\right)$ and $\bar{S}_{C}^{(i)}\left(\boldsymbol{q}, \omega_{q} ; \omega_{l}\right)(i=a, b)$ always have terms with the factor $\left(i \omega_{l}+1 / \tau\right)^{-2}$ and $\left(i \omega_{l}+1 / \tau\right)^{-1}$ when $i=c]$, as shown in Appendix G4. Moreover, in any case of chiral fluctuations, the expression for the chirality-dependent fluctuation propagator, (B3), holds besides numerical factors. Therefore, it is general that $\alpha_{x y \text { chiral }}^{\text {Kubo }}$ and $\sigma_{x y \text { chiral }}$ are proportional to $\tau^{2}$ in the clean limit. However, their magnitudes depend on the functional form of $W$ as will be discussed in Appendix G3.

\section{Critical behavior}

We discuss how the critical exponents of $\alpha_{x y \text { chiral }}^{\text {Kubo }}$ and $\sigma_{x y \text { chiral }}$ as functions of $\varepsilon=\log T / T_{c}$ depend on the functional form of $W\left(\boldsymbol{k}, \omega_{j}\right)$, the dimensionality, and the pairing symmetry of chiral superconducting states.

As mentioned above, we can use the expression of the chirality-dependent fluctuation propagator (B3) generally ex-
TABLE I. Critical behavior of $\alpha_{x y \text { chiral }}^{\text {Kubo }}$ and $\sigma_{x y \text { chiral }}$, which is determined by the dimensionality and the pairing symmetry of chiral superconducting states.

\begin{tabular}{lcc}
\hline \hline & \multicolumn{2}{c}{$\Delta$} \\
\cline { 2 - 3 } & 0 & 2 \\
\hline $2 \mathrm{D}$ & $1 / \varepsilon$ & $\log \varepsilon$ \\
& $\left(\mathrm{Sr}_{2} \mathrm{RuO}_{4}\right)$ & \\
$3 \mathrm{D}$ & $1 / \sqrt{\varepsilon}$ & const. $-\sqrt{\varepsilon}$ \\
& & $\left(\mathrm{URu}_{2} \mathrm{Si}_{2}\right)$ \\
\hline \hline
\end{tabular}

cept numerical factors. Then, from Eq. (B45), we obtain

$$
\alpha_{x y \text { chiral }}^{\text {Kubo }} \text { or } \sigma_{x y \text { chiral }} \propto \int d^{d} \boldsymbol{q} \frac{q^{\Delta}}{\left(\xi^{2} q^{2}+\varepsilon\right)^{2}}
$$

in the vicinity of $T_{c}$, where $\Delta$ is defined by $\bar{A}_{C}\left(\boldsymbol{q}, \omega_{q}=\right.$ $\left.0 ; \omega_{l}\right)$ or $\bar{S}_{C}\left(\boldsymbol{q}, \omega_{q}=0 ; \omega_{l}\right)=O\left(q^{\Delta}\right)$, as $q \rightarrow 0$. Therefore we find that the spatial dimensionality and $\Delta$ determine the critical exponent.

As will be shown in Sec. G 4, $\Delta$ does not depend on specific forms of $W\left(\boldsymbol{k}, \omega_{j}\right)$, but is affected by the spatial dimensionality and pairing symmetries of chiral superconducting states. Therefore, the critical exponents of (4) and (5) are independent of the specific form of $W\left(\boldsymbol{k}, \omega_{j}\right)$, though the dimensionality may change them.

In Table I, we present the exponents for some typical examples. In the case of the three-dimensional chiral $d_{z x} \pm i d_{z y}$ superconducting fluctuation, $\Delta=2$ [see Eqs. (B30)-(B33)], and then the critical behavior is $\alpha_{x y \text { chiral }}^{\text {Kubo }} \propto$ const. $-\sqrt{\varepsilon}$ [see Eq. (3)(3)(3)(4)]. Moreover, in the case of the two-dimensional chiral $p+i p$ superconductor [the $d$-vector is given by $\boldsymbol{d}(\boldsymbol{k})=\left(0,0, k_{x} \pm i k_{y}\right)$, and then $\phi(\boldsymbol{k}) \propto\left(k_{x} \pm i k_{y}\right)$, which is believed to be the paring symmetry of $\mathrm{Sr}_{2} \mathrm{RuO}_{4}$ [15], we find, from straightforward calculations, $\bar{A}_{C}, \bar{S}_{C} \propto q^{0}$ and then $\alpha_{x y \text { chiral }}^{\text {Kubo }}, \sigma_{x y \text { chiral }} \propto 1 / \varepsilon$, where the critical behavior of the former (latter) is the same as (less singular than) that of the conventional AL term in two spatial dimensions [30].

\section{Specific form of $W$}

In this subsection, we discuss how the magnitudes of $\alpha_{x y \text { chiral }}^{\text {Kubo }}$ and $\sigma_{x y \text { chiral }}$ are influenced by the momentum dependence of $W$.

Here we consider the case of arbitrary chiral paring symmetry that is given by $\phi(\boldsymbol{p}) \propto Y_{l}^{m}(\hat{p})$ (three dimension) or $\propto e^{i m \theta_{p}}$ (two dimension), where $Y_{l}^{m}$ is the spherical harmonic function and $\theta_{\boldsymbol{p}}$ is the angle defined by $\tan \theta_{\boldsymbol{p}}=p_{x} / p_{y}$. As discussed in Appendix $\mathrm{B}$, only the odd parts of $\bar{A}_{C}$ or $\bar{S}_{C}$ with respect to time-reversal operation, $C \rightarrow-C$, give nonzero contributions. The integrands of $\bar{A}_{C=1}^{(i)}-\bar{A}_{C=-1}^{(i)}$ and $\bar{S}_{C=1}^{(i)}-\bar{S}_{C=-1}^{(i)}$ generally $(i=a, b, c)$ contain the factor (see Fig. 5)

$$
\begin{aligned}
& \phi^{\dagger}(\boldsymbol{p}) \phi\left(\boldsymbol{p}^{\prime}\right) W\left(\boldsymbol{p}-\boldsymbol{p}^{\prime}, \omega_{j}\right)-\left(\phi \leftrightarrow \phi^{\prime}\right) \\
& \propto \sin (m \theta) W\left(\boldsymbol{p}-\boldsymbol{p}^{\prime}, \omega_{j}\right),
\end{aligned}
$$

where $\theta$ is the angle between $\boldsymbol{p}_{\|}$and $\boldsymbol{p}_{\|}^{\prime}$. Here, $\boldsymbol{p}_{\|}\left(\boldsymbol{p}_{\|}^{\prime}\right)$ represents the projection of the vector $\boldsymbol{p}\left(\boldsymbol{p}^{\prime}\right)$ onto the $a b$ 


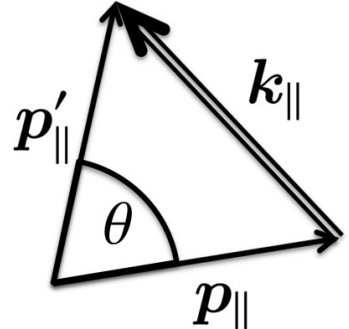

FIG. 7. Relation between the relative momentums of fluctuating Cooper pairs, $\boldsymbol{p}, \boldsymbol{p}^{\prime}$, the momentum of four-point vertex, $\boldsymbol{k}$, and the angle $\theta$ that is the angle between $\boldsymbol{p}_{\|}$and $\boldsymbol{p}_{\|}^{\prime}$.

plane. The relation between these vectors and angle is drawn in Fig. 7. From this equation, we find that, due to the factor $\sin (m \theta)$, the magnitudes of $\alpha_{x y \text { chiral }}^{\text {Kubo }}$ and $\sigma_{x y \text { chiral }}$ are large in the case that the magnitude of $W\left(\boldsymbol{p}-\boldsymbol{p}^{\prime}, \omega_{j}\right)$ is large for $\theta \sim$ (half odd integer) $\times(\pi / m)$.

We now consider a simple case that the momentum dependence of $W\left(\boldsymbol{k}, \omega_{j}\right)$ has a dominant peak at $\boldsymbol{k}=\boldsymbol{Q}_{0}$ with width $1 / \xi_{Q_{0}}$. When the peak is sharp, the domain of integration that contributes to $\alpha_{x y \text { chiral }}^{\text {Kubo }}$ and $\sigma_{x y \text { chiral }}$ is restricted to the region in which $\boldsymbol{p}-\boldsymbol{p}^{\prime} \sim \boldsymbol{Q}_{0}$. However, it is quite exceptional that the angle $\theta$ is nearly equal to (half odd integer) $\times(\pi / m)$ when $\boldsymbol{p}$ and $\boldsymbol{p}^{\prime}$ satisfy the above condition. Therefore, generally, the magnitudes of $\alpha_{x y \text { chiral }}^{\text {Kubo }}$ and $\sigma_{x y \text { chiral }}$ are small when $\xi_{\boldsymbol{Q}_{0}}$ is large. On the other hand, when $\xi_{Q_{0}}$ is small, the domain of integration in which $\theta \sim$ (half odd integer) $\times(\pi / m)$ is included in the domain in which $W\left(\boldsymbol{p}-\boldsymbol{p}^{\prime}, \omega_{j}\right)$ has large values, and then the magnitudes of $\alpha_{x y \text { chiral }}^{\text {Kubo }}$ and $\sigma_{x y \text { chiral }}$ are larger than the case of large $\xi_{\boldsymbol{Q}_{0}}$. As a result, the magnitudes of $\alpha_{x y \text { chiral }}^{\text {Kubo }}$ and $\sigma_{x y \text { chiral }}$ increase as $\xi_{Q_{0}}$ become smaller.

Finally, we consider the case of $\mathrm{URu}_{2} \mathrm{Si}_{2}$. In this case, the pairing function is given by $\phi(\boldsymbol{p}) \propto Y_{2}^{1}(\hat{p})$ and the interaction is mediated via short-range antiferromagnetic spin fluctuation with $\boldsymbol{Q}_{0}=\left(0,0,2 \pi / a_{z}^{\text {lattice }}\right)$, where $a_{z}^{\text {lattice }}$ is the lattice constant. Here $\xi_{Q_{0}}$ is the correlation length of this fluctuation. Since $\theta$ is zero when $\boldsymbol{Q}_{0}=\boldsymbol{p}-\boldsymbol{p}^{\prime}$, then the magnitudes of $\alpha_{x y \text { chiral }}^{\text {Kubo }}$ and $\sigma_{x y \text { chiral }}$ become small, if this fluctuation were to be long range.

\section{Auxiliary explanations}

a. Factors $\left(i \omega_{l}+1 / \tau\right)^{-2}$ and $\left(i \omega_{l}+1 / \tau\right)^{-1}$ in $\bar{A}_{C}^{(i)}\left(q, \omega_{q} ; \omega_{l}\right)$ and

$$
\bar{S}_{C}^{(i)}\left(q, \omega_{q} ; \omega_{l}\right)
$$

Here, we show that in any case of the functional form of $W$, the dimensionality, and the pairing symmetry of chiral superconducting states, $\bar{A}_{C}^{(i)}\left(\boldsymbol{q}, \omega_{q} ; \omega_{l}\right)$ and $\bar{S}_{C}^{(i)}\left(\boldsymbol{q}, \omega_{q} ; \omega_{l}\right)$ for $i=a, b$ always have the terms proportional to $\left(i \omega_{l}+1 / \tau\right)^{-2}$ [and $\left(i \omega_{l}+1 / \tau\right)^{-1}$ for $i=c$.

First, for $i=a$, in any case, we can write [see Fig. 5(a)]

$$
\begin{aligned}
\bar{A}_{C}^{(a)} & \left(\boldsymbol{q}, \omega_{q} ; \omega_{l}\right) \text { or } \bar{S}_{C}^{(a)}\left(\boldsymbol{q}, \omega_{q} ; \omega_{l}\right) \\
= & \sum_{\boldsymbol{p}, \boldsymbol{s}, \boldsymbol{q}, n, m} G\left(\boldsymbol{p}, \varepsilon_{n-l}\right) G\left(\boldsymbol{p}, \varepsilon_{n}\right) G\left(\boldsymbol{s}, \varepsilon_{m-l}\right) G\left(\boldsymbol{s}, \varepsilon_{m}\right) \\
& \times g^{a}(\boldsymbol{p}, \boldsymbol{s}, n, m ; l),
\end{aligned}
$$

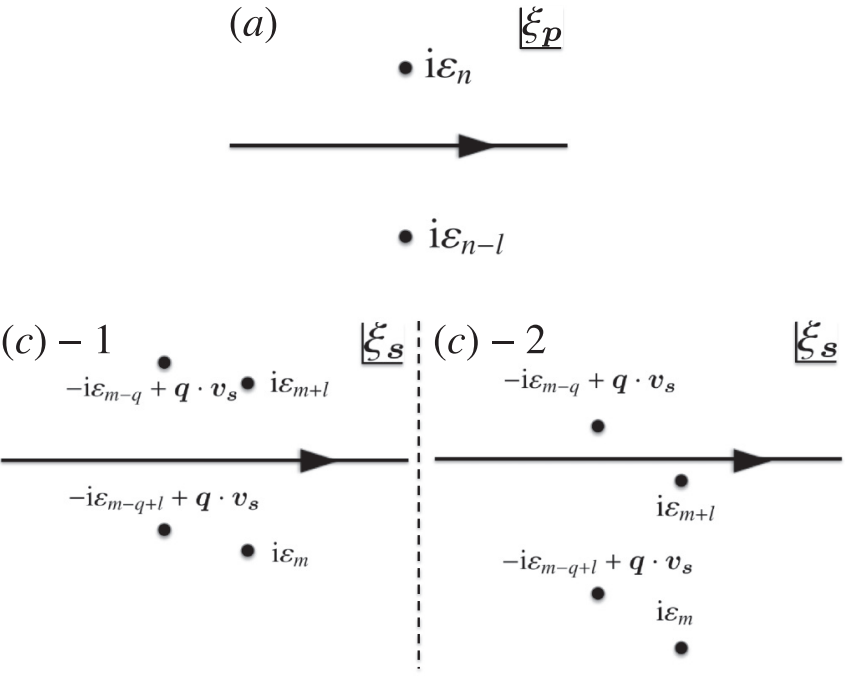

FIG. 8. The contours of energy integration in Eqs. (G3) (upper panel) and (G4) (lower panels). Dots represent poles of order 1. Each contour integration generates the factor $\left(i \omega_{l}+1 / \tau\right)^{-1}$.

where $g^{a}$ is a certain function. Then, integrating it over the energy $\xi_{p}$ along the contour shown in Fig. 8(a), we obtain the factor $\left(i \varepsilon_{n}-i \varepsilon_{n-l}\right)^{-1}=\left(i \omega_{l}+1 / \tau\right)^{-1}$ at $n=0,1, \ldots, l-1$, and this factor does not appear for other values of $m$. Similarly, the integration over $\xi_{s}$ also generates the factor $\left(i \omega_{l}+\right.$ $1 / \tau)^{-1}$ at $m=0,1, \ldots, l-1$. Therefore, $\bar{A}_{C}^{(a)}\left(\boldsymbol{q}, \omega_{q} ; \omega_{l}\right)$ and $\bar{S}_{C}^{(a)}\left(\boldsymbol{q}, \omega_{q} ; \omega_{l}\right)$ have terms with factor $\left(i \omega_{l}+1 / \tau\right)^{-2}$. We can prove this relation also for the case of $i=b$ by using a similar argument.

Next, for $i=c$, we can write [see Fig. 5(c)]

$$
\begin{aligned}
\bar{A}_{C}^{(c)}\left(\boldsymbol{q}, \omega_{q} ; \omega_{l}\right) \text { or } \bar{S}_{C}^{(c)}\left(\boldsymbol{q}, \omega_{q} ; \omega_{l}\right) \\
=\sum_{\boldsymbol{s}, \boldsymbol{p}, m, n} G\left(\boldsymbol{s}, \varepsilon_{m+l}\right) G\left(\boldsymbol{s}, \varepsilon_{m}\right) G\left(\boldsymbol{q}-\boldsymbol{s},-\varepsilon_{m-q}\right) \\
\quad \times G\left(\boldsymbol{q}-\boldsymbol{s},-\varepsilon_{m-q+l}\right) G\left(\boldsymbol{q}-\boldsymbol{p},-\varepsilon_{n-q+l}\right) \\
\quad \times G\left(\boldsymbol{p}, \varepsilon_{n+l}\right) g^{c}(\boldsymbol{s}, \boldsymbol{p}, \boldsymbol{q}, m ; l),
\end{aligned}
$$

where $g^{c}$ is a certain function. When $\max \{-l, q-l\} \leqslant m \leqslant$ $\min \{-1, q-1\}$, the pole structure of the complex $\xi_{s}$ plane is given by Fig. 8(c), and then integrating over $\xi_{s}$ generates the terms proportional to $\left(i \omega_{l}+1 / \tau\right)^{-1}$. Such terms appear also for the value of $m$, at which three poles exist in the upper half plane or lower half plane and the other one exits in the other side [Fig. 8(c)-2], and do not appear when all poles exist on the same side. On the other hand, integrating over $\xi_{p}$ generates no term proportional to $\left(i \omega_{l}+1 / \tau\right)^{-1}$. Therefore, $\bar{A}_{C}^{(c)}\left(\boldsymbol{q}, \omega_{q} ; \omega_{l}\right)$ and $\bar{S}_{C}^{(c)}\left(\boldsymbol{q}, \omega_{q} ; \omega_{l}\right)$ have terms with a factor $\left(i \omega_{l}+1 / \tau\right)^{-1}$.

\section{b. Critical exponent $\Delta$ independent of specific form of $W$}

In this subsection, we show that the critical exponent $\Delta$ is independent of the specific form of $W\left(\boldsymbol{k}, \omega_{j}\right)$. We consider the case that the interaction is repulsive, i.e., $W\left(\boldsymbol{k}, \omega_{j}\right)>0$. 
For every $i=a, b, c$, we can write $\bar{A}_{C}^{(i)}$ or $\bar{S}_{C}^{(i)}$ as

$$
\begin{gathered}
\bar{A}_{C}^{(i)}\left(\boldsymbol{q}, \omega_{q}=0 ; \omega_{l}\right) \text { or } \bar{S}_{C}^{(i)}\left(\boldsymbol{q}, \omega_{q}=0 ; \omega_{l}\right) \\
=\sum_{\boldsymbol{k}, \omega_{j}} h\left(\boldsymbol{q}, \boldsymbol{k}, \omega_{j} ; \omega_{l}\right) W\left(\boldsymbol{k}, \omega_{j}\right) .
\end{gathered}
$$

The important point is that $W$ is independent of $\boldsymbol{q}$. Then, expanding $h\left(\boldsymbol{q}, \boldsymbol{k}, \omega_{j} ; \omega_{l}\right)$ with respect to $\boldsymbol{q}$, we obtain

$$
\begin{aligned}
& \bar{A}_{C}^{(i)}\left(\boldsymbol{q}, \omega_{q}=0 ; \omega_{l}\right) \text { or } \bar{S}_{C}^{(i)}\left(\boldsymbol{q}, \omega_{q}=0 ; \omega_{l}\right) \\
& =\sum_{\boldsymbol{k}, \omega_{j}} h\left(\boldsymbol{k}, \omega_{j} ; \omega_{l}\right) W\left(\boldsymbol{k}, \omega_{j}\right)+q_{x}^{2} \sum_{\boldsymbol{k}, \omega_{j}} h_{x x}\left(\boldsymbol{k}, \omega_{j} ; \omega_{l}\right) W\left(\boldsymbol{k}, \omega_{j}\right) \\
& \quad+q_{y}^{2} \sum_{\boldsymbol{k}, \omega_{j}} h_{y y}\left(\boldsymbol{k}, \omega_{j} ; \omega_{l}\right) W\left(\boldsymbol{k}, \omega_{j}\right)+\cdots .
\end{aligned}
$$

Here, we neglect terms that vanish after performing the integration, (G1). $\Delta$ is the lowest number of dimensions with respect to $\boldsymbol{q}$ of the term in which $\sum h_{*} W$ is nonzero, for $*=x x, y y, z z, x x x x, x x y y, \ldots$. Since $W$ is positive definite, $\sum h_{*} W$ leads to $\sum h_{*} W^{\prime}=0$, where $W^{\prime}$ is another form of potential energy that is positive definite. Therefore, $\Delta$ for some particular form of $W$ is the same as that of another one, $W^{\prime}$, and thus we conclude that $\Delta$ is independent of the specific form of $W$.

\section{APPENDIX H: BERRY PHASE FLUCTUATION}

In this section, we discuss a possible relation between the chirality-fluctuation-induced anomalous Nernst (Hall) effect and the Berry phase. Chiral superconductors generally possess the nonzero Berry curvature below $T_{c}$. Because of the nonzero Berry curvature, $\mathrm{Sr}_{2} \mathrm{RuO}_{4}$, which is believed to be a quasi-2D $p+i p$ superconductor, is regarded as a candidate of a class $\mathrm{D}$ topological superconductor, and also, $\mathrm{URu}_{2} \mathrm{Si}_{2}$, which is supposed to be a 3D $d+i d$ superconductor, is a candidate of a Weyl superconductor. We speculate that the ANE and AHE considered in this paper may be deeply related to the Berry phase inherent in chiral superconductors. In fact, the chiral superconducting order parameters should always accompany the nonzero Berry curvature, which implies that even above $T_{c}$, chiral superconducting fluctuations are related to the Berry curvature fluctuation. Furthermore, our scenario is also applicable to the Rashba $s$-wave superconductor with the strong Zeeman field, in which the superconducting order parameter is nonchiral, but the nonzero Berry curvature exists below $T_{c}$ [48]. This implies that the most important factor of our mechanism of the ANE and AHE is not chiral superconducting fluctuation, but rather, the nontriviality of fluctuations associated with the Berry curvature, which may be referred to as "Berry phase fluctuation." In the following subsections, we discuss these points.

\section{Berry curvature of chiral superconductors in the vicinity of $T_{c}$}

In this subsection, we discuss the relation between the Berry curvature and chiral superconducting fluctuations. For simplicity, we consider a 2D spinless chiral $p+i p$ superconductor, the Bogoliubov-de Gennes Hamiltonian of which is given by

$$
H_{p+i p}=\left(\begin{array}{cc}
\xi_{k} & \Delta\left(k_{x}-i k_{y}\right) \\
\Delta\left(k_{x}+i k_{y}\right) & -\xi_{k}
\end{array}\right),
$$

where $\Delta|\boldsymbol{k}|$ is the gap amplitude, and $\xi_{\boldsymbol{k}}=\frac{|\boldsymbol{k}|^{2}}{2 m}-\mu$. The Berry curvature of this system in the momentum space $\Omega_{k_{x} k_{y}}$ is

$$
\Omega_{k_{x} k_{y}}=\frac{\xi_{k} \Delta^{2}}{2\left(\xi_{k}^{2}+\Delta^{2}|\boldsymbol{k}|^{2}\right)^{3 / 2}} .
$$

Although this expression is applicable only to the mean-field Hamiltonian without fluctuation below $T_{c}$, we formally put $\Delta=\Delta_{0}+\delta \Delta$ with $\Delta_{0}$ a static part and $\delta \Delta$ a fluctuating part in Eq. (H2), and consider the limit of $\Delta_{0} \rightarrow 0$. Then, for small $\delta \Delta$ and $\boldsymbol{k}$ away from the Fermi surface, we have

$$
\Omega_{k_{x} k_{y}}=\frac{(\delta \Delta)^{2}}{2 \xi_{k}^{2}} .
$$

Note that the right-hand side of Eq. (H3) is nonzero even above $T_{c}$ when there is a Gaussian superconducting fluctuation $\left\langle(\delta \Delta)^{2}\right\rangle \neq 0$. It should be cautioned that Eq. (H3) does not mean the nonzero Berry curvature above $T_{c}$. In fact, the static Berry curvature is zero above $T_{c}$. We, rather, call the quantity defined by Eq. (H3) the Berry phase (or curvature) fluctuation. The above relation implies that the chiral superconducting fluctuation may induce the Berry phase fluctuation even above $T_{c}$. This indicates a possible relation between the Berry phase fluctuation and the anomalous Nernst (Hall) effect raised by chiral superconducting fluctuation.

\section{Relation between Rashba $s$-wave superconductor and chiral $p+i p$ superconductor}

In this subsection, we first prove that the Rashba $s$ wave superconductor is mapped to the chiral $p_{x}+i p_{y}$-wave superconductor when the Zeeman field is sufficiently large.

The model of the Rashba $s$-wave superconductor with the gauge filed $A_{\mu}$ and gravitational field $\phi_{g}$, which causes the thermal current, is given by

$$
\begin{aligned}
H_{\phi_{g}, A_{\mu}}^{\text {Rashba }}= & \int d^{2} r\left(1+\phi_{g}\right)\left[\psi ^ { \dagger } \left(-\frac{\nabla^{2}}{2 m}-\mu-e A_{0}\right.\right. \\
& \left.\left.-i \lambda \sigma \cdot \nabla-\mu_{B} H_{z} \sigma^{z}\right) \psi-g \psi_{\uparrow}^{\dagger} \psi_{\downarrow}^{\dagger} \psi_{\downarrow} \psi_{\uparrow}\right],
\end{aligned}
$$

where $\psi=\left(\psi_{\uparrow}, \psi_{\downarrow}\right)^{t} ; \nabla_{i}=\partial_{i}-i e A_{i}$ for $i=x, y ; \mu$ is the chemical potential; $\mu_{B} H_{z}>0$ is the Zeeman energy; and $g>0$ is the coupling constant of the effective attractive interaction in the $s$-wave channel. We assume that the chemical potential is sufficiently weaker than the Zeeman energy, $|\mu| \ll \mu_{B} H_{z}$, such that there is only one Fermi surface. Here, the electron field in the band crossing the Fermi surface is denoted as $\Psi_{-}$and the one in its orthogonal band $\Psi_{+}$. When the Zeeman field is sufficiently strong as $\mu_{B} H_{z} \gg \lambda k_{F}$, we obtain $\psi_{\uparrow}=\Psi_{-}+\left[i \lambda\left(\nabla_{z}-i \nabla_{y}\right) / \mu_{B} H_{z}\right] \Psi_{+}$and $\psi_{\downarrow}=$ $\left[-i \lambda\left(\nabla_{z}+i \nabla_{y}\right) / \mu_{B} H_{z}\right] \Psi_{-}+\Psi_{+}$. Therefore, neglecting the electrons $\Psi_{+}$, whose band does not cross the Fermi level, 
Eq. (H4) is rewritten as

$$
\begin{aligned}
H_{\phi_{g}, A_{\mu}}^{\text {Rashba }} \cong & \int d^{2} r\left(1+\phi_{g}\right)\left[\Psi_{-}^{\dagger}\left(-\frac{\nabla^{2}}{2 m}-\mu_{\text {eff }}-e A_{0}\right) \Psi_{-}\right. \\
& -\tilde{g}\left\{V^{p+i p}\left(\frac{-i\left(\partial_{1}-\partial_{2}\right)}{2}, \frac{-i\left(\partial_{1}^{\prime}-\partial_{2}^{\prime}\right)}{2}\right)\right. \\
& \left.\left.\Psi_{-}^{\dagger}\left(\boldsymbol{r}_{1}\right) \Psi_{-}^{\dagger}\left(\boldsymbol{r}_{2}\right) \Psi_{-}\left(\boldsymbol{r}_{2}^{\prime}\right) \Psi_{-}\left(\boldsymbol{r}^{\prime}{ }_{1}\right)\right\}_{\boldsymbol{r}_{1}, \boldsymbol{r}_{2}, \boldsymbol{r}_{1}^{\prime}, \boldsymbol{r}_{2}^{\prime} \rightarrow \boldsymbol{r}}\right],
\end{aligned}
$$

where $V^{p+i p}\left(\boldsymbol{k}, \boldsymbol{k}^{\prime}\right)=\left(k_{x}+i k_{y}\right)\left(k_{x}^{\prime}-i k_{y}^{\prime}\right), \tilde{g}=g\left(\lambda / \mu_{B} H_{z}\right)^{2}$, and $\mu_{\text {eff }}=\mu+\mu_{B} H_{z}$. This is the model of the spineless chiral $p_{x}+i p_{y}$-wave superconductor.
Thus, if there are additional scattering processes raised by an effective four-point vertex $W\left(\boldsymbol{k}, \omega_{j}\right)$, we can apply our theory to this emergent chiral $p+i p$ superconductor, and obtain the ANE and AHE. However, in this case, the effects are raised not by chiral superconducting fluctuations but by nonchiral $s$-wave superconducting fluctuations of the original system (H4), which induces "effective chirality." We note that the origin of the "effective chirality" is essentially related to the existence of the nonzero Berry curvature below $T_{c}$ for the Rashba $s$-wave superconductor with the strong Zeeman field [48], though the Berry curvature is zero above $T_{c}$ within the mean-field theory. Thus, we speculate that the most important and indispensable factor which raises the ANE and AHE considered in this paper is not the chiral superconducting fluctuation, but rather the nontriviality of fluctuations associated with the Berry phase, and that the Berry phase fluctuation may play an important role in our scenario.
[1] Z. Xu, N. Ong, Y. Wang, T. Kakeshita, and S. Uchida, Nature (London) 406, 486 (2000).

[2] A. Pourret, H. Aubin, J. Lesueur, C. A. Marrache-Kikuchi, L. Bergé, L. Dumoulin, and K. Behnia, Nat. Phys. 2, 683 (2006).

[3] K. Behnia, J. Phys.: Condens. Matter 21, 113101 (2009).

[4] Note that there are some metallic systems such as bithmus for which the Sondheimer cancellation does not occur. This is due to the large mobility and the small Fermi energy associated with specific band structures [3].

[5] I. Ussishkin, S. Sondhi, and D. A. Huse, Phys. Rev. Lett. 89, 287001 (2002).

[6] Y. Wang, Z. A. Xu, T. Kakeshita, S. Uchida, S. Ono, Y. Ando, and N. P. Ong, Phys. Rev. B 64, 224519 (2001).

[7] It is almost proven that the origin of the enhanced Nernst effect above and near $T_{c}$ of dirty superconducting films is the shortlived Cooper pair [2]. Also in the case of high- $T_{c}$ cuprates, recent experimental studies strongly support this scenario $[49,50]$.

[8] A. Furusaki, M. Matsumoto, and M. Sigrist, Phys. Rev. B 64, 054514 (2001).

[9] R. Roy and C. Kallin, Phys. Rev. B 77, 174513 (2008).

[10] J. Xia, Y. Maeno, P. T. Beyersdorf, M. M. Fejer, and A. Kapitulnik, Phys. Rev. Lett. 97, 167002 (2006).

[11] N. Read and D. Green, Phys. Rev. B 61, 10267 (2000).

[12] K. Nomura, S. Ryu, A. Furusaki, and N. Nagaosa, Phys. Rev. Lett. 108, 026802 (2012).

[13] H. Sumiyoshi and S. Fujimoto, J. Phys. Soc. Jpn. 82, 023602 (2013).

[14] N. Nagaosa, J. Sinova, S. Onoda, A. H. MacDonald, and N. P. Ong, Rev. Mod. Phys. 82, 1539 (2010).

[15] A. P. Mackenzie and Y. Maeno, Rev. Mod. Phys. 75, 657 (2003).

[16] Y. Kasahara, T. Iwasawa, H. Shishido, T. Shibauchi, K. Behnia, Y. Haga, T. D. Matsuda, Y. Onuki, M. Sigrist, and Y. Matsuda, Phys. Rev. Lett. 99, 116402 (2007).

[17] Y. Kasahara, H. Shishido, T. Shibauchi, Y. Haga, T. D. Matsuda, Y. Onuki, and Y. Matsuda, New J. Phys. 11, 055061 (2009).
[18] K. Yano, T. Sakakibara, T. Tayama, M. Yokoyama, H. Amitsuka, Y. Homma, P. Miranović, M. Ichioka, Y. Tsutsumi, and K. Machida, Phys. Rev. Lett. 100, 017004 (2008).

[19] R. Nandkishore, L. S. Levitov, and A. V. Chubukov, Nat. Phys. 8, 158 (2012)

[20] F. Liu, C.-C. Liu, K. Wu, F. Yang, and Y. Yao, Phys. Rev. Lett. 111, 066804 (2013).

[21] P. K. Biswas, H. Luetkens, T. Neupert, T. Stürzer, C. Baines, G. Pascua, A. P. Schnyder, M. H. Fischer, J. Goryo, M. R. Lees, et al., Phys. Rev. B 87, 180503 (2013).

[22] M. H. Fischer, T. Neupert, C. Platt, A. P. Schnyder, W. Hanke, J. Goryo, R. Thomale, and M. Sigrist, Phys. Rev. B 89, 020509(R) (2014).

[23] M. L. Kiesel, C. Platt, W. Hanke, and R. Thomale, Phys. Rev. Lett. 111, 097001 (2013).

[24] R. Okazaki, Y. Kasahara, H. Shishido, M. Konczykowski, K. Behnia, Y. Haga, T. D. Matsuda, Y. Onuki, T. Shibauchi, and Y. Matsuda, Phys. Rev. Lett. 100, 037004 (2008).

[25] J. M. Luttinger, Phys. Rev. 135, A1505 (1964).

[26] Yu. N. Obraztsov, Sov. Phys. Solid State 6, 331 (1964); 7, 455 (1965).

[27] L. Smrcka and P. Streda, J. Phys. C 10, 2153 (1977).

[28] N. R. Cooper, B. I. Halperin, and I. M. Ruzin, Phys. Rev. B 55, 2344 (1997).

[29] M. Sigrist and K. Ueda, Rev. Mod. Phys. 63, 239 (1991).

[30] A. Larkin and A. Varlamov, Theory of Fluctuations in Superconductors (Oxford University Press, 2009).

[31] J. Schwinger, Phys. Rev. 82, 664 (1951).

[32] M. Khodas and A. M. Finkel'stein, Phys. Rev. B 68, 155114 (2003).

[33] K. Michaeli, K. S. Tikhonov, and A. M. Finkel'stein, Phys. Rev. B 86, 014515 (2012).

[34] E. Helfand and N. R. Werthamer, Phys. Rev. 147, 288 (1966).

[35] F. Bourdarot, B. Fåk, K. Habicht, and K. Prokeš, Phys. Rev. Lett. 90, 067203 (2003). 
[36] C. R. Wiebe, J. A. Janik, G. J. MacDougall, G. M. Luke, J. D. Garrett, H. D. Zhou, Y.-J. Jo, L. Balicas, Y. Qiu, J. R. D. Copley, et al., Nat. Phys. 3, 96 (2007).

[37] V. M. Galitski and A. I. Larkin, Phys. Rev. B 63, 174506 (2001).

[38] I. Ussishkin, Phys. Rev. B 68, 024517 (2003).

[39] M. Serbyn, M. Skvortsov, A. Varlamov, and V. Galitski, Phys. Rev. Lett. 102, 067001 (2009).

[40] K. Michaeli and A. M. Finkel'stein, Europhys. Lett. 86, 27007 (2009).

[41] A. G. Aronov, S. Hikami, and A. I. Larkin, Phys. Rev. B 51, 3880 (1995)

[42] N. A. Sinitsyn, A. H. MacDonald, T. Jungwirth, V. K. Dugaev, and J. Sinova, Phys. Rev. B 75, 045315 (2007).

[43] T. Yamashita, S. Tonegawa, Y. Tsuruhara, Y. Shimoyama, T. Shibauchi, and Y. Matsuda (private communication).
[44] F. Rullier-Albenque, R. Tourbot, H. Alloul, P. Lejay, D. Colson, and A. Forget, Phys. Rev. Lett. 96, 067002 (2006).

[45] Y. Sidis, M. Braden, P. Bourges, B. Hennion, S. NishiZaki, Y. Maeno, and Y. Mori, Phys. Rev. Lett. 83, 3320 (1999).

[46] W. Schlabitz, J. Baumann, B. Pollit, U. Rauchschwalbe, H. Mayer, U. Ahlheim, and C. Bredl, Z. Phys. B 62, 171 (1986).

[47] P. G. de Gennes, Superconductivity of Metals and Alloys, 2nd ed. (Addison-Wesley, Reading, MA, 1989).

[48] M. Sato, Y. Takahashi, and S. Fujimoto, Phys. Rev. Lett. 103, 020401 (2009).

[49] J. Chang, N. Doiron-Leyraud, O. Cyr-Choiniere, G. Grissonnanche, F. Laliberte, E. Hassinger, J.-P. Reid, R. Daou, S. Pyon, T. Takayama, et al., Nat. Phys. 8, 751 (2012).

[50] F. F. Tafti, F. Laliberté, M. Dion, J. Gaudet, P. Fournier, and L. Taillefer, Phys. Rev. B 90, 024519 (2014). 